\title{
Hybrid System: Parallel Neural -Genetic Algorithm Algorithm for Compacting Fractal Images Using Multiple Computers
}

\author{
Shahla A. Abdel-Qader Suzan K. Ibrahim Omia Gh. Abdel-Jabbar \\ Computer Systems Department \\ Technical Institute / Mosul, Iraq
}

Received on: 09/09/2012

Accepted on: 30/01/2013

\begin{abstract}
Recently, effective technologies in Fractal Image Coding (FIC) were used to reduce the complexity of search for the matching between the Range blocks and the Domain blocks which reduces the time needed for calculation. The aim of this research is to propose a Hybird Parallel Neural -Genetic Algorithm (HPNGA) using the technique of (Manager/Worker) in multiple computers in order to obtain the fastest and best compression through extracting the features of the gray and colored images to attenuate the problem of dimensions in them .The NN enabled to train separate images from the test images to reduce the calculation time. The $\mathrm{NN}$ able to adapt itself with the training data to reduce the complexity and having more data and is merged with the parallel GA to reach optimum values of weights with their biases. The optimum weights obtained will classify the correct search domains with the least deviation, which, in turn ,helps decompress the images using the fractal method with the minimum time and with high resolution through multiple computers. The results showed that the proposed hybrid system is faster than the standard algorithm, the NN and GA in decompressing the FIC and they are flexible and effective to reach the optimum solution with high speed and resolution. The search method used for compression and de-compression has a vital role in improving the ratio and the quality of image compression which reached $15_{\mathrm{s}}$.The ratio of compression reached to $90.68 \%$ and the image improvement after decompression reached to $34.71_{\mathrm{db}}$ when compared to other methods of (FIC), which didn't exceed $90.41 \%$ and image quality of $32.41_{\mathrm{db}}$ and the execution speed was only $21_{\text {s. }}$.
\end{abstract}

Keywords: Hybird System, Artificial Neural Network(ANN), Parallel Genetic Algorithm (PGA), Fractal Image Coding(FIC), Rang Block, Domain Block.

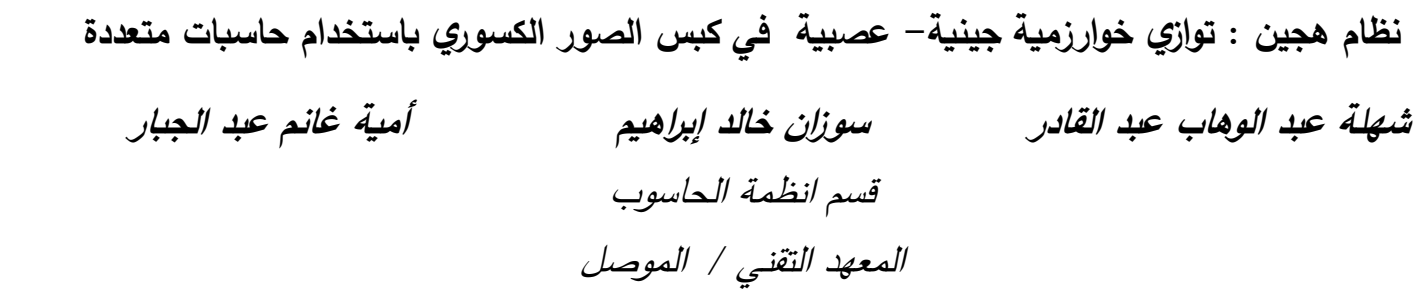

تاريخ قبول البحث: 2013/01/30

تاريخ استلام البحث: 2012/09/09

\section{الملخص}

استخدمت مؤخرا تقنيات فعالة في التشفير الكسوري للصور Fractal Image Coding(FIC) لتقليل

تعقيد البحث للتطابق بين كتل المدى مange Block وكتل المجال Domain Block الذي يقلل الوقت المستغرق في الحساب. الهدف من هذا البحث اقتراح نظام هجين : توازي خوارزمية جينية- عصبية في كبس 
الصور الكسوري Hybird Parallel Neural -Genetic Algorithm (HPNGA بتقنية المدير/العامل في حاسبات متعددة للحصول على اسرع وافضل كبس من خلال إستخلاص الصفات للصور الرمادية والملونة لتقليل مشكلة الأبعاد فيها، اذ مكنت الثبكة العصبية من تدريب صور منفصلة عن صور الاختبار لتقليل وقت الحساب،

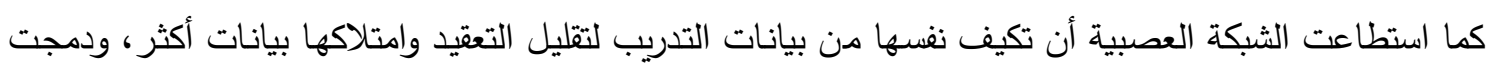

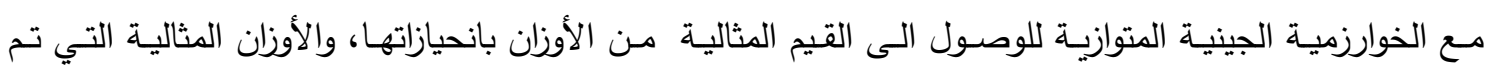

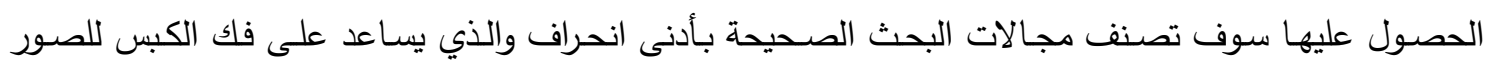

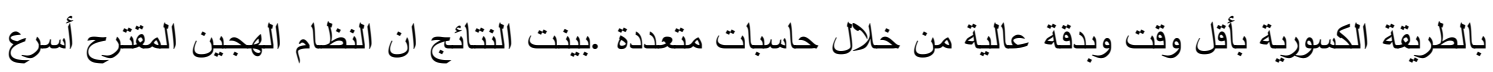

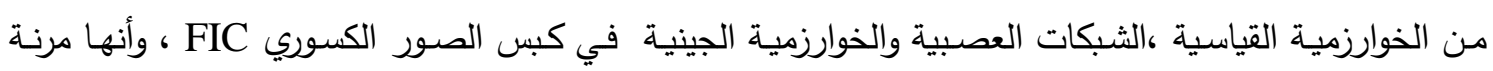
وفعالة للوصول إلى الحل الأمثل بسرعة وكفاءة عاليتين، وان طريقة البحث المستخدمة للكبس وفك الكبس كان لها

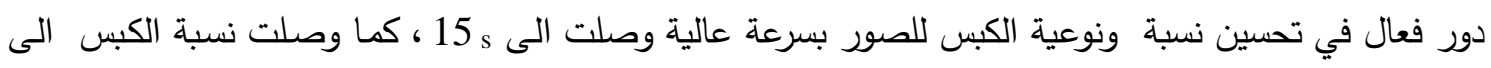
\% 90.68 وتحسين نوعية الصورة بعد فك الكبس الى ما يقارب db 34.71مقارنة بالطرائق الاخرى لكبس الصور

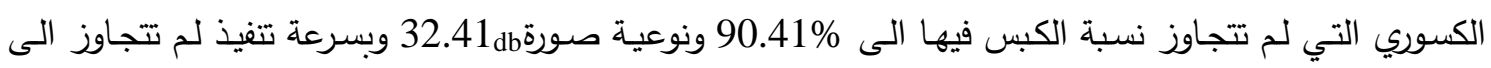
$.21_{\mathrm{s}}$

الكلمـات المفتاحيـة: النظام الهجين، الثبكات العصبية الاصطناعية، الخوارزمية الجينية المتوازيـة، كبس الصور الكسوري، كتلة المدى، كتلة المجال.

1 - المقدمة

كبس وفك الكبس للصور من تقنيات الصور الرقمية التي أصبحت جانبا مهما في خزنها ونقلها في مجال

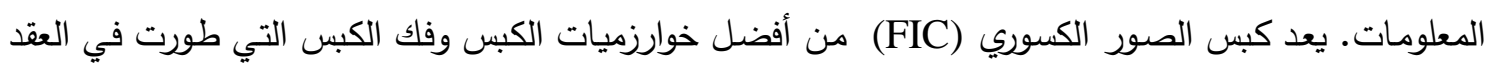

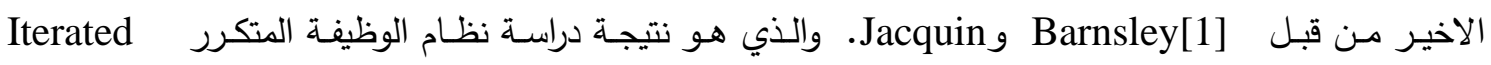
Function System (IFS) compression ratio

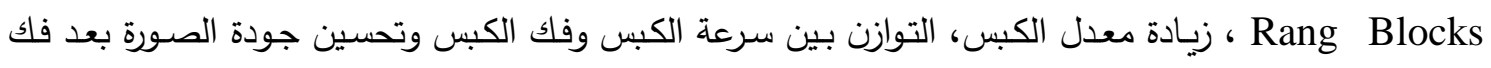

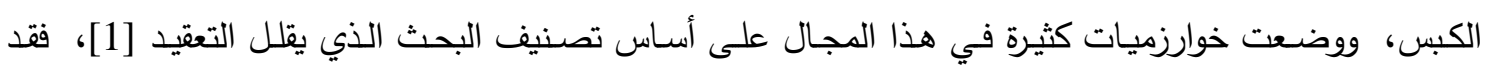
استخدم باحثون خوارزميات التقنيات الذكائية مثل (ANN,GA) لكبس الصور الكسوري. الخوارزميات الجينية تعتبرمن تقنيات البحث الذكي التي تُستخدم لإيجاد حلول تقريبية للمسائل التي تتطلب

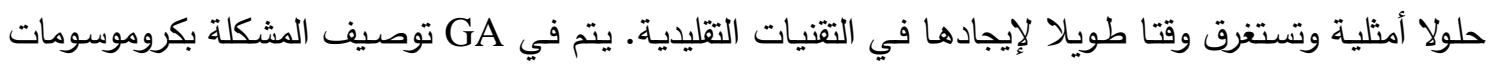

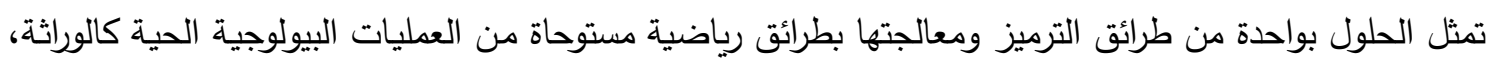

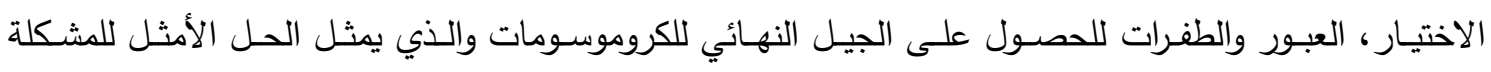

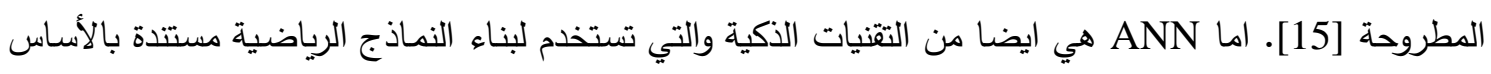

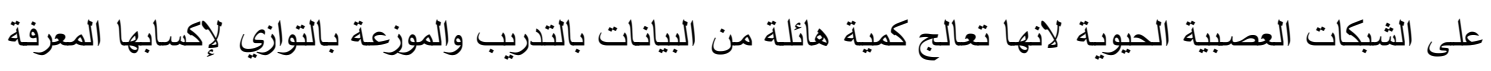
لغرض التطبيق ،والمعالجة في ANN تتم من خلال وحدات معالجة تسمى عقد Node والتي لها خاصية عصبية

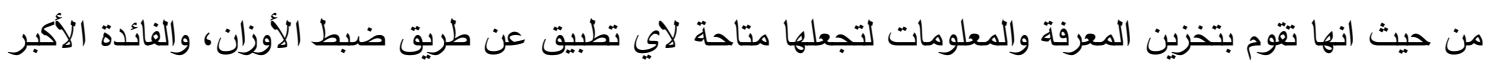


في ANN هي قدرتها على تثكيل العلاقات اللاخطية المعقدة بدون فرضيات استتتاجيه عن طبيعة العلاقة][2] لذلك استخدمت GA و GNN لتصنيف مجال البحث عن كتل المدى وكتل المجال في كبس الصور الكسوري. الباحث M.Mohamed [3],2006 عمل على تطوير NN لكبس الصور الكسوري، فيما عمل الباحثون M.Sheng ,Y.Chakrapani [6],2009, X.Lifeng[5],2007, A.Ben [4],2006

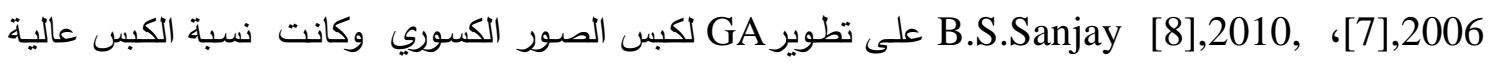

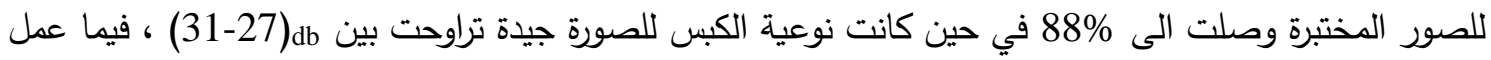

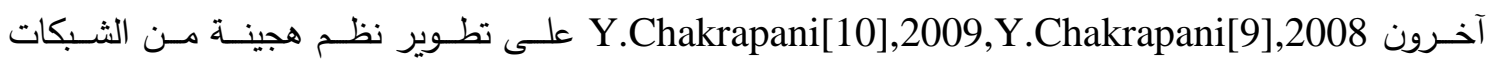
العصبية والمنطق المضبب لكبس الصور الكسوري وقد كانت النتائج متفاوتة بين طريقة واخرى فقد وصلت نسبة

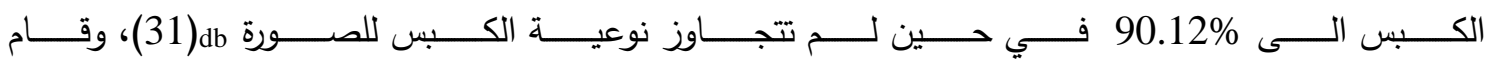
الباحثونY.Chakrapani[12],2009 ,Y.Chakrapani[11],2008 بتطبيق خوارزمية جينية-عصبية لتسريع

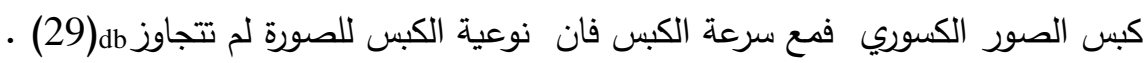

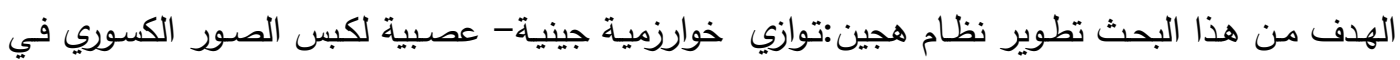

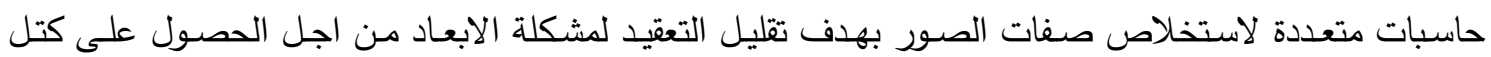

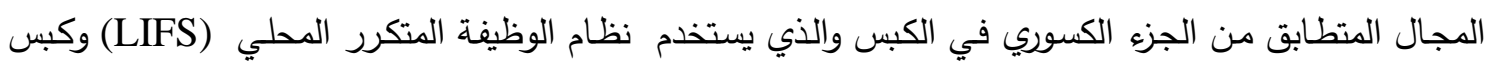
الصور الكسوري للصور الرمادية والملونة التي تقلل الوقت وتحسن نوعية الصورة بعد فك الكبس.

2- توازي خوارزمية جينية - عصبية لكبس الصور الكسوري ستراتيجية النظام الهجين في كبس الصور الكسوري تكون كالاتي:1-2 كبس الصور الكسوري (FIC):

كبس الصورة الكسوري عملية معقدة جدا لكن فك الكبس عملية سهلة جدا مما يجعل معدل الكبس عاليا" فيها، هذا التعقيد جاء لانها تستند بالاساس على نظريـة الوظيفة المتكرر LISF. التعقيد في كبس صورة هورة أصلية بطريقة FIC هو كيفية الحصول على معلمات LIFS، وكالاتي:-

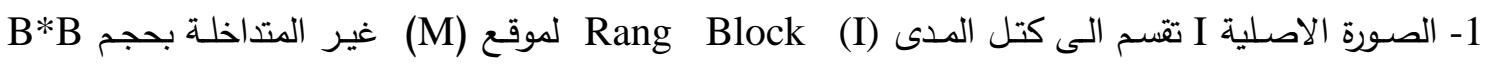
يوضحها الثكل (1) ، وبشكل عشوائي لموقع (N) لكتل المجال Domain Blocks(I) وبحجم 2B*2B. كتل

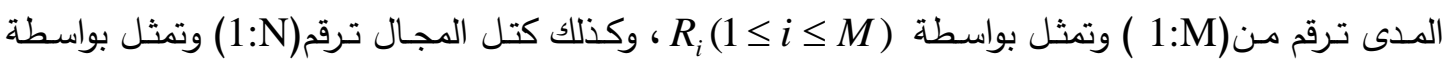
. $D_{j}(1 \leq j \leq N)$

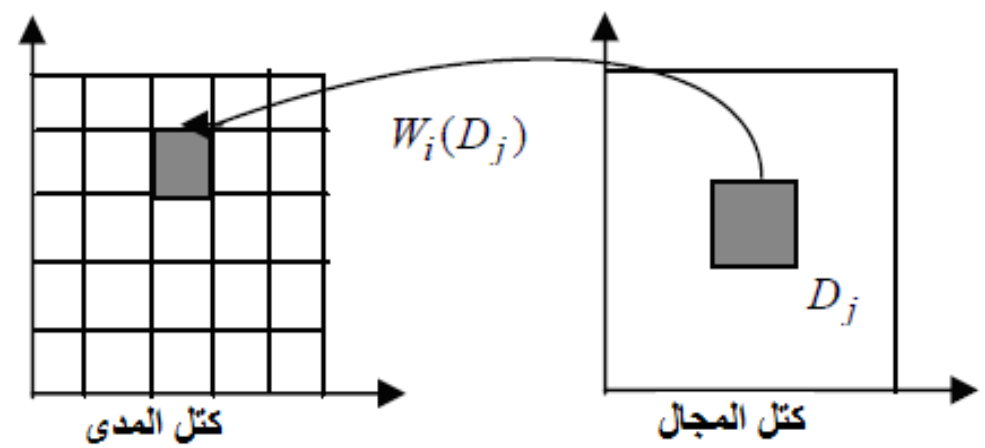

الثكل رقم (1): تحولات كتل المدى -المجال 


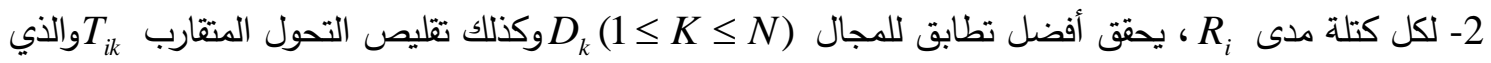
يحقق بالمعادلة التالية[5] :

$d\left(R_{i}, T_{i k}\left(D_{k}\right)\right)=\min . d\left(R_{i}, T_{i j}\left(D_{j}\right)\right)$

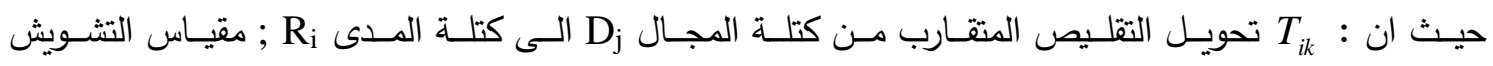

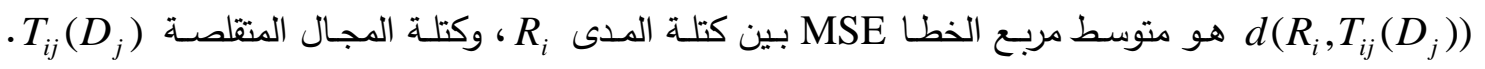

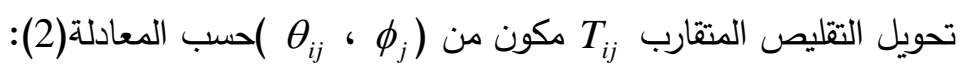

$T_{i j}=\theta_{i j} \circ \phi_{j}$

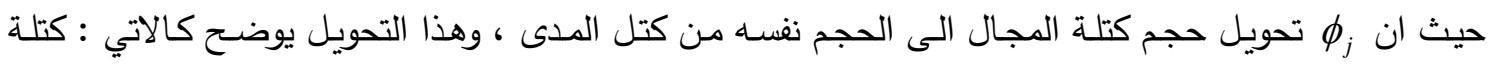
المجال D تقسم الى وحدات غير متداخلة بحجم 2*2 2 , وكل نقطة صورية pixel في كتلة التحويل

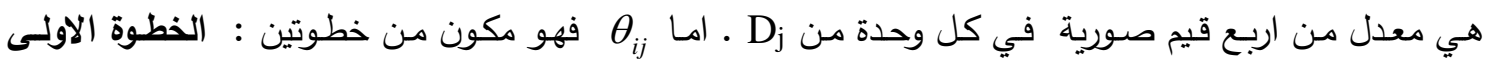
تحولات كتلـة (D) للارجات (270+,

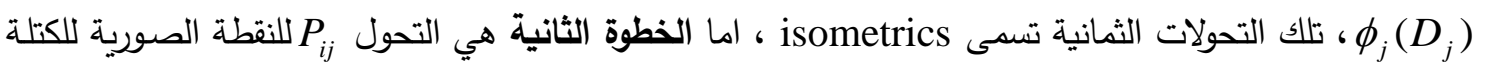

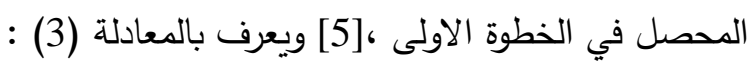
$P_{i j}(v)=a_{i j} v+g_{i j}$

حيث ان : هي النقطة الصورية pixel للكتلة الناتجة في الخطوة الاولى .

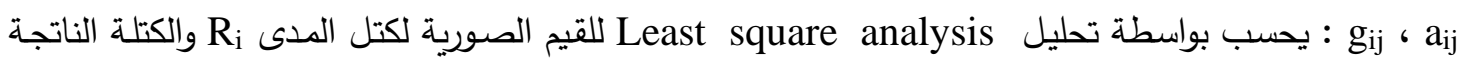
بواسطة الخطوة الاولى. ويسمى aij : معامل القياس ، ويسمى gij : معامل التعديل .المشكلة الأساسية في هذه الطريقة هو استهلاك وقت كبير في FIC، في هذا البحث طور نظام هجين توازي خوارزمية جينية -عصبية في

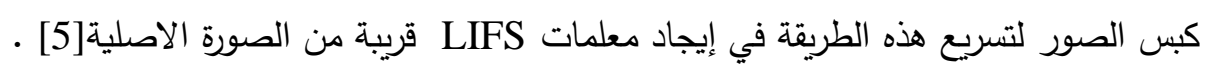

Features Extraction 2-2 استخلاص الصفات

كبس الصور الكسوري يستغرق وقتا طويلا لأنها تحتاج الى عدد كبير من حسابات التطابق بين كتل المدى ،

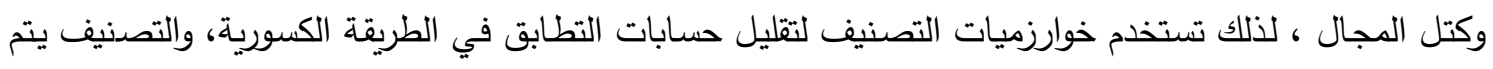
في هذا البحث باستخدام ANN وبخوارزمية الانتشار العكسي التي تدرب على بيانات الصفات المستخلصـة من

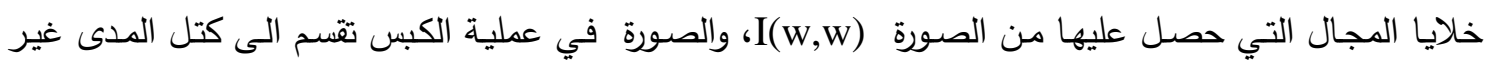

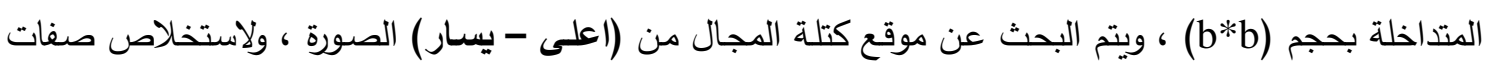

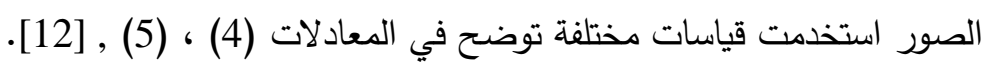
1- Standard Deviation (

$$
\sigma=\sqrt{\frac{1}{n_{r} n_{c}} \sum_{i=1}^{n_{r}} \sum_{j=1}^{n_{c}}\left(P_{i, j}-M\right)^{2}}
$$

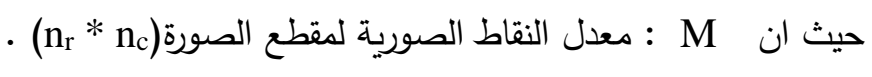

· P : Pi,j

Skewness -2 : الذي يعطى بالمعادلة الاتية [12]: 


$$
S k=\frac{1}{n_{r} n_{c}} \sum_{i=1}^{n_{r}} \sum_{j=1}^{n_{c}} \frac{\left(P_{i, j}-M\right)^{3}}{\sigma^{3}}
$$

Multi Computer 3-2 الحاسبات المتوازية

هو شكل من أشكال الحوسبة التي يجري فيها تتفيذ العديد من العمليات في وقت واحد والتي تعمل من مبدأ ان المشاكل الكبيرة يمكن ان تقسم على مشاكل اصغر حجما ويتم حلها في وقت متزامن بالتوازي بحيث يكون

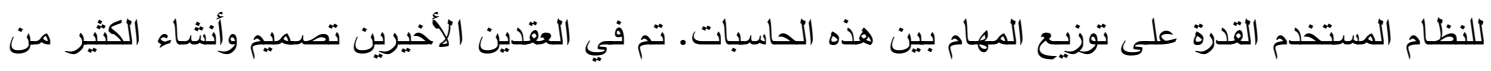

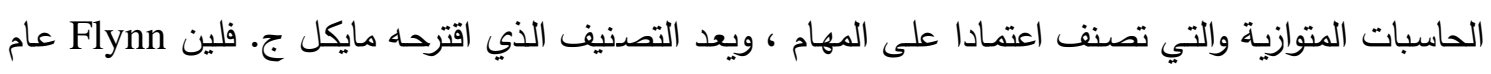

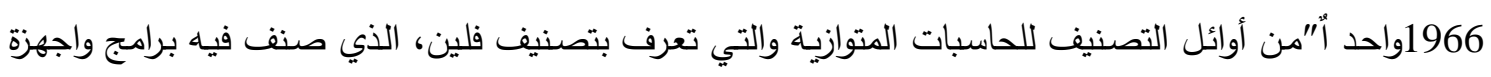

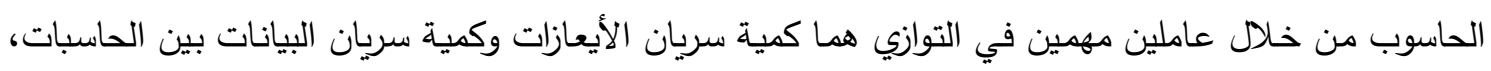

$$
\text { وعليه فان فلاين صنف الحاسبات[16] كالاتي : }
$$

1-الحاسبات وحيدة الايعازات ووحيدة البيانات والذي يعني برنامج متسلسل تماما 2. Data(SISD) كبيرة من البيانات وهو شائع الاستعمال(Single Instruction Multiple Data(SIMD). 3-الحاسبات متعددة

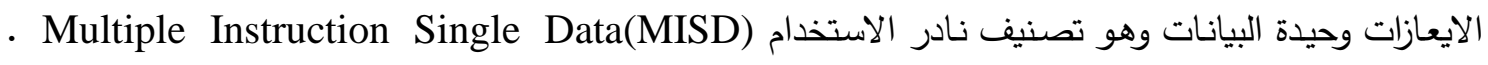

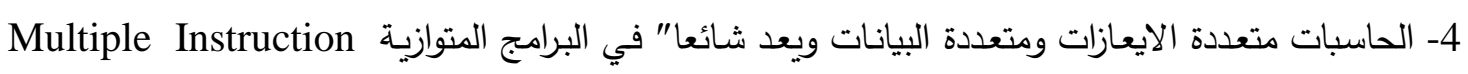
.Multiple Data(MIMD)

استخدم في هذا البحث التصنيف Single Program Multiple Data(SPMD) المطور عن التصنيف والذي يعني توازيا"على مستوى المهام بحيث يحقق سريان الايعازات ببرنامج واحد الى الحاسبات المتوازيـة

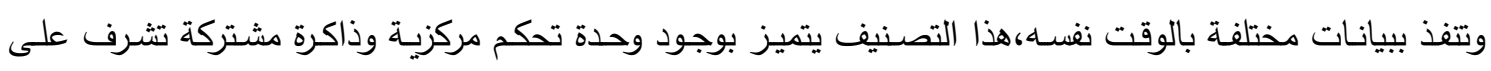

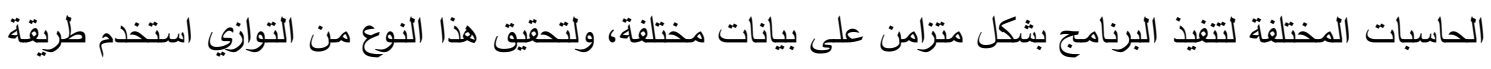

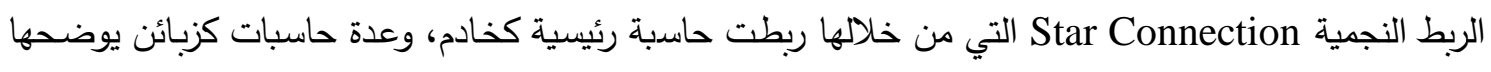

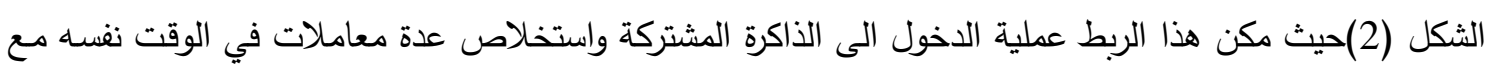

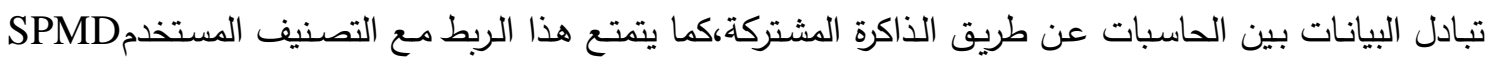

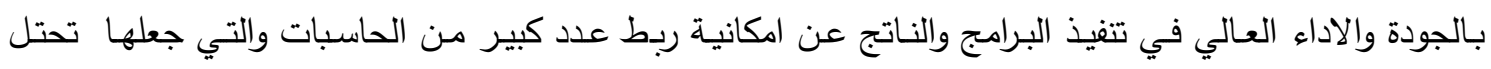

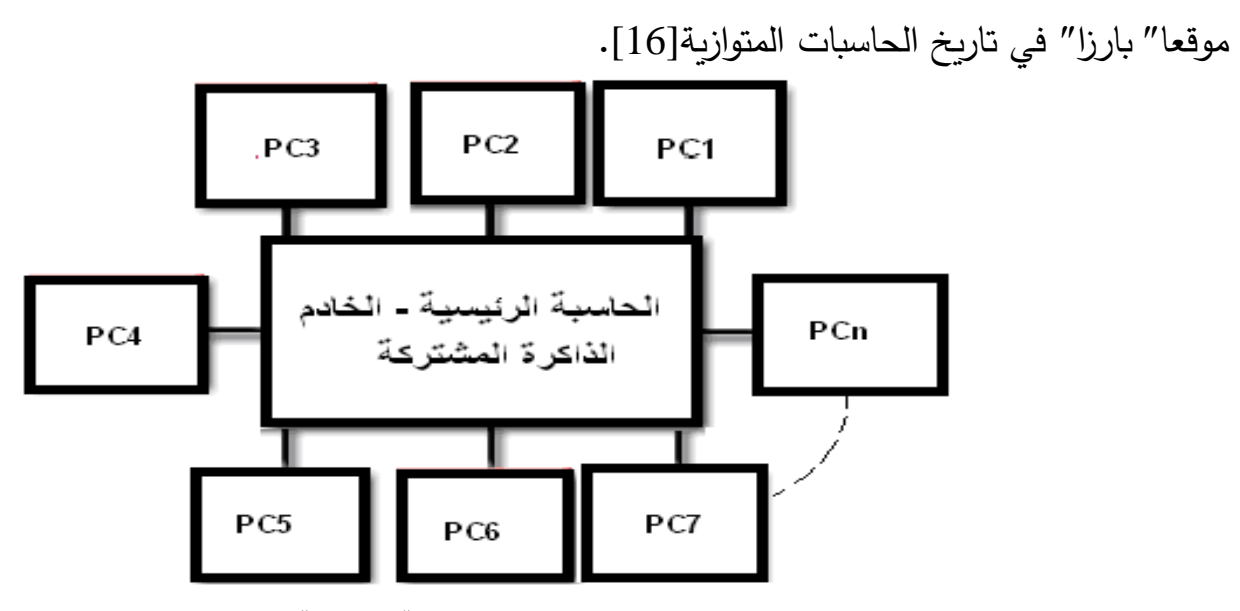

الثكل رقم (2): الثبكة النجمية 


\section{ANN 4-2 الثبكة العصبية الاصطناعية}

في حقل كبس الصور طورت الكثير من البحوث التي تستخدم التقنيات الذكائية مثل ANN لكبس وفك

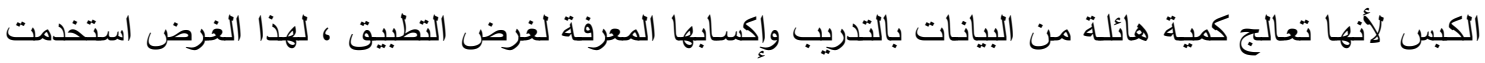

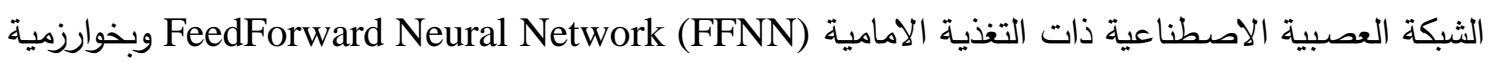
تدريب ذات انتشار عكسي لتصنيف النقط الصورية للمجال ،والثبكة مكونة من عدة طبقات : طبقة الادخال، طبقة الاخراج وواحدة او اكثر من الطبقات الخفية، ولا تحتوي على روابط رجوع بصورة عكسية ،الإخراج يحسب مباشرة

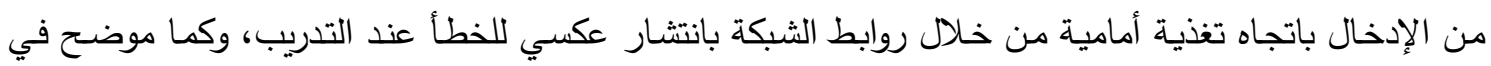

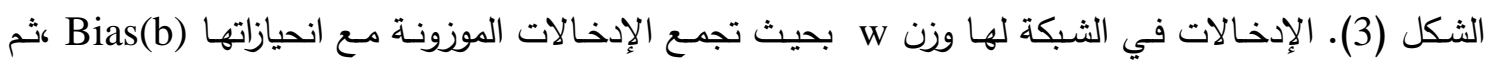
تطبق عليها دالة التتشيط Activation Function (AF) والتي يمكن ان يستعمل أنواع منها لتوليد الإخراج

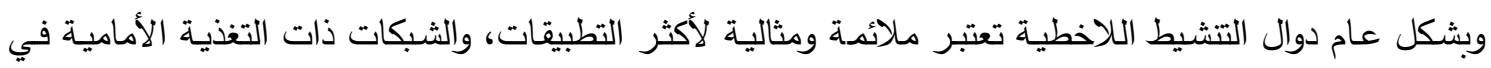

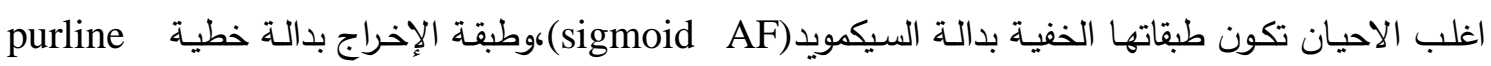

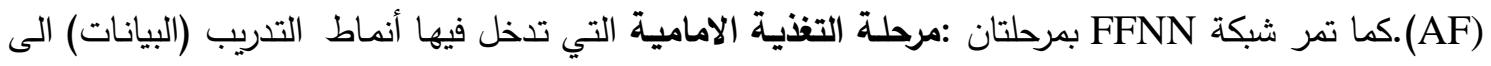

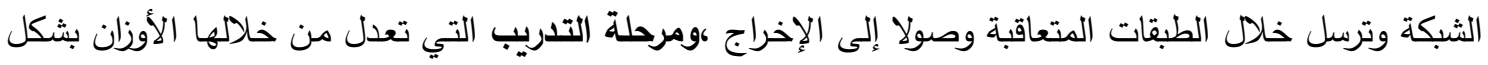
تكراري لكل أنماط التدريب على أساس تقليل الأخطاء بين مخرجات الثبكة والمخرجات الحقيقة بانحدار تدريجي

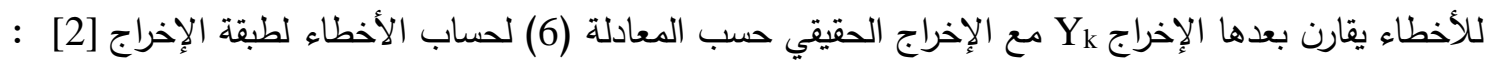
$\delta_{k}=\left(T_{k}-Y_{k}\right) * Y_{k}\left(1-Y_{k}\right)$

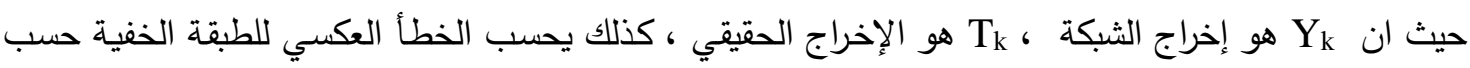

المعادلة (7):

$\delta_{k}=Y_{k}\left(1-Y_{k}\right) \sum \delta_{k} * W_{k}$

حيث ان d o هو معدل الخطا بين طبقة الاخراج والطبقة الخفية ، في حين تعدل أوزان الثبكة في كل مرحلة من

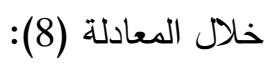

$W_{j k}(t+1)=W_{j k}(t)+\eta \delta_{k} Y_{k}+\alpha\left(W_{j k}(t)-W_{j k}(t-1)\right)$

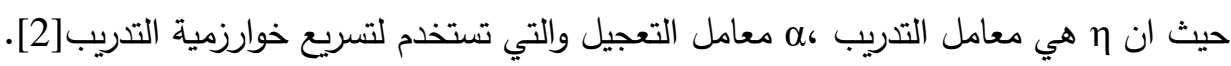

FIC GA 5-2 الخوارزميات الجينية لكبس الصور الكسوري

السمة الرئيسية لـFIC هو إيجاد كتلة المجال المناسبة والتحول إلى نوعية من كتلة المدى ، والمشكلة فيها

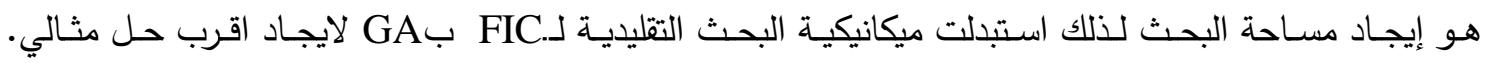

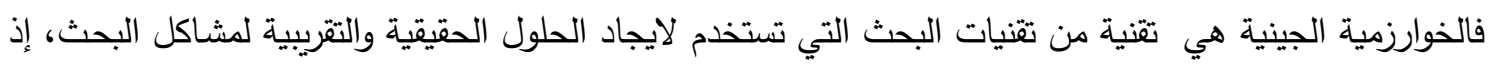

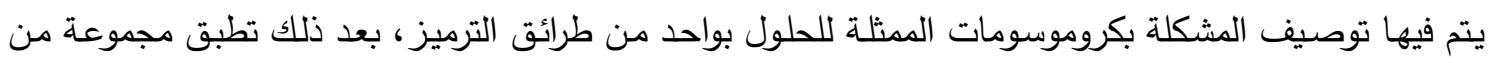

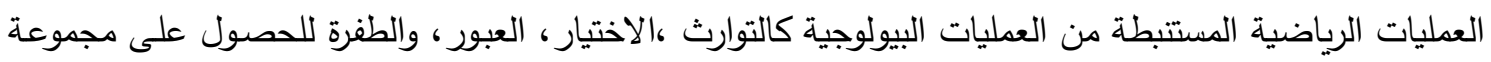

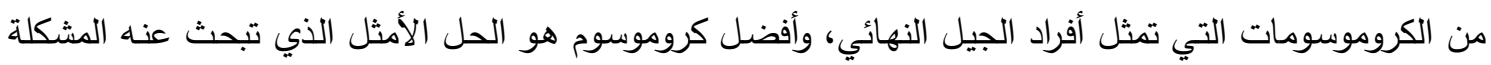

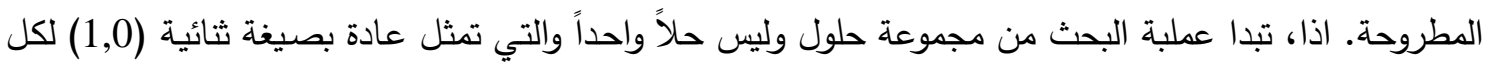
الاحتمالات الممكنة للمسألة، والجيل الاول يبدا بتوليد جيل عشوائي لكل الكروموسومات التي تمثل مساحة الحل. 
الجيل الجديد يتكون من تقييم كل فرد في الجيل القديم حسب دالة اللياقة، استخدمت هذه الطريقة في كبس الصور

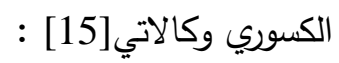

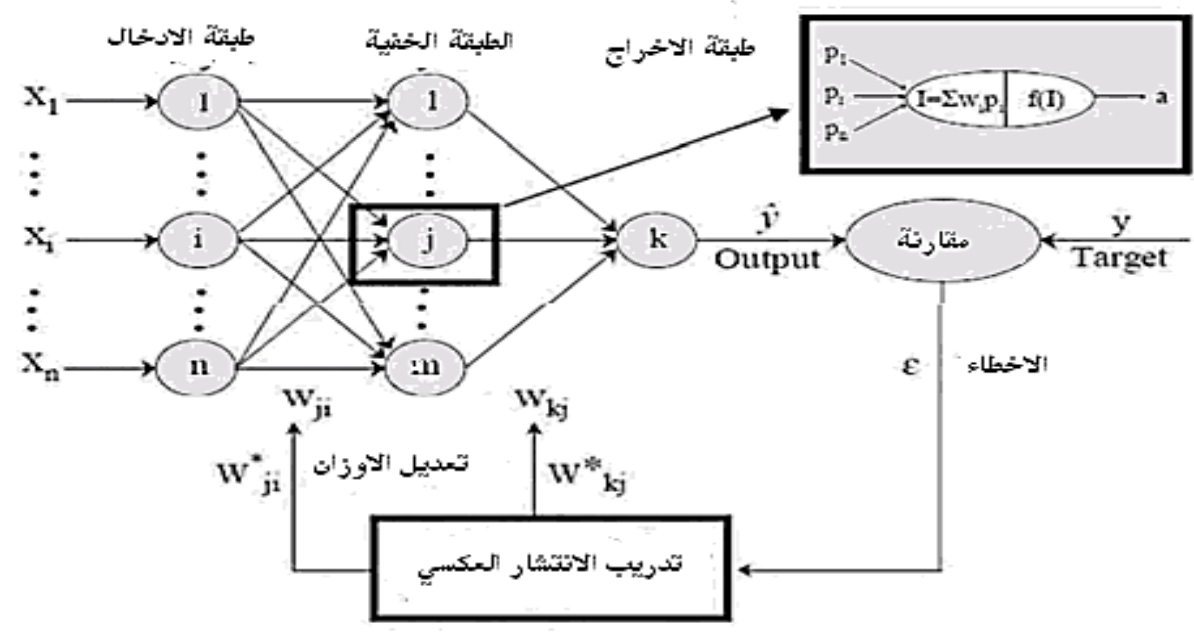

الثكل رقم (3): نموذج مثالي لثبكة الاتتشار العكسي

تشفيرالمعلماتParameter coding:بـابصورة ثنائية والتي تعتمد على قيم

الاوزان ومعامل الانحياز ـANN للبحث عن كتلة المجال يوضحها الثكل (4) ـ

\begin{tabular}{|c|c|c|c|c|c|c|}
\hline 11010 & ....... & 11010 & 11010 & ......... & 11010 & 11010 \\
\hline $\mathrm{V}_{11}$ & ....... & $\mathrm{V}_{23}$ & $\mathrm{~W}_{11}$ & $\ldots \ldots \ldots$ & $\mathrm{W}_{31}$ & B \\
\hline \multicolumn{3}{|c|}{$\leftarrow$ Input Weight $\rightarrow$} & \multicolumn{3}{|c|}{$\leftarrow$ Output Weight $\rightarrow$} & Bias \\
\hline
\end{tabular}

الثكل (4): الكرموسوم للشبكة العصبية الاصطناعية

• حجم الجيل Population size : يقصد به عدد الأفراد ضمن الجيل وهو من العوامل المهمة التي يتوقف

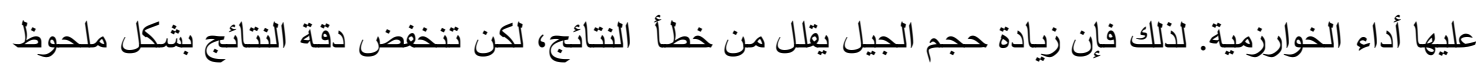
عند كون حجم الجيل صغيرًا لأنه لن يشمل كامل المجال المدروس بشكل جيد ،وتتم عملية بناء الجيل الابتدائي initial population • دالة اللياقة Fitness Function: سلسلة الكروموسومات المكونة للجيل القديم من بيانات الصورة تتغير الى لى $F i(x)=\left\{\begin{array}{l}\frac{1}{m s e(x)+1} \ldots m s e(x)>\varepsilon \\ \infty \ldots \ldots \ldots \ldots \ldots \ldots . . . m s e(x) \leq \varepsilon\end{array}\right\}$

الجيل الجديد اعتمادا على دالة اللياقة بالمعادلة (9) : حيث MSE(X) : هو متوسط مربع الخطأ بين كتلة المدى وكتلة المجال المحصلة من الكروموسوم X ، وان

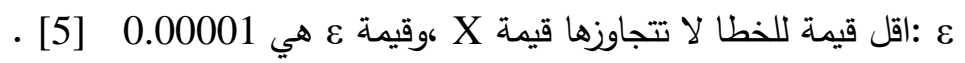

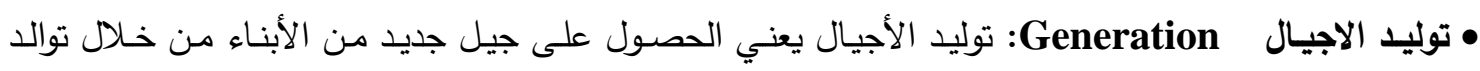

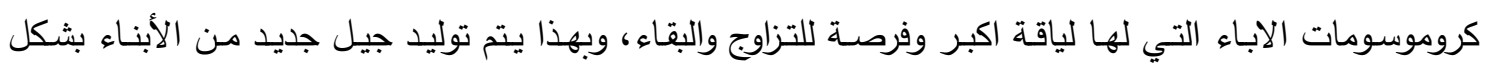

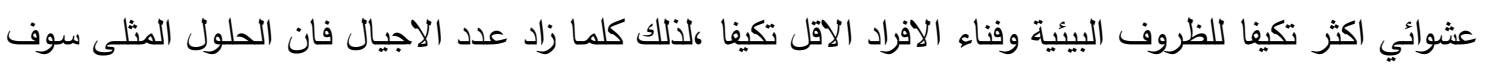
تحافظ على نفسها في الاجيال اللاحقة باقل تقدير، وتمر عملية التوليد بثلاث مراحل، ( الاختيار ، العبور، الطفرة). 
• الاختيار على وفق مبدا العجلة المتدحرجة Roulette Wheel Selection: محاكاة هذه الطريقة تتم بوجود عجلة لها مؤشر تقسم إلى 100 قطاع ،أفراد الجيل يُوزَّعون على القطاعات وفقًا لمتوسط احتمالية الانتخاب لكل فرد

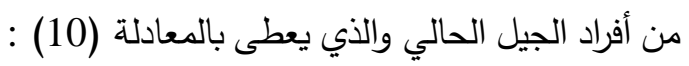

Pis $=\frac{F i}{\left(\sum_{i=1}^{m} F i\right)},(i=1,2, \ldots \ldots \ldots . M)$

حيث أن : Pis هي احتمالية انتخاب الفرد Fi , i : درجة لياقة الفرد i ، و M و : عدد الأجيال.

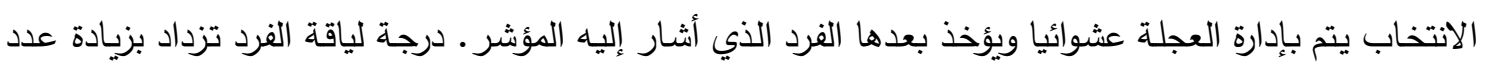
قطاعاته ومن ثم يزاد أحتمال انتخابه في الجيل اللاحق بتأثير إيجابي.

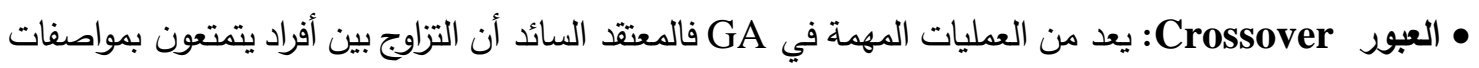

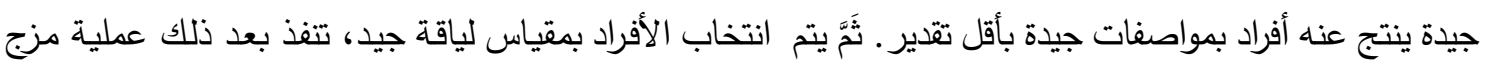

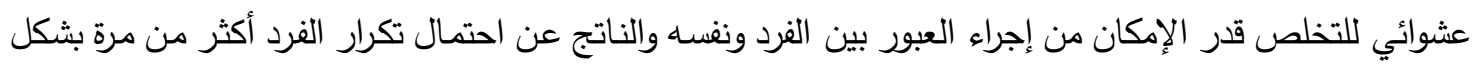
متتالٍ نتيجة عملية الاختيار التي تسبق العبور ،تتم عملية العبور بين الفرد والذي يليه باستخدام إحدى طرائق التئق العبور

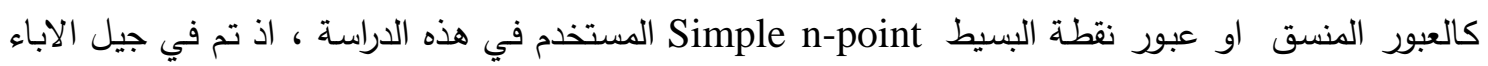
تقسيم الكروموسومات الى (مجموعتين) والعبور يتم فيها عشوائيا لكل مجموعة وفق احتمالية العبور of Crossover(Pc)

\begin{tabular}{|c|c|c|c|c|c|c|c|}
\hline \multirow{2}{*}{ جيل الاباء } & $V_{1,1}$ & $V_{1,2}$ & $V_{1,3}$ & $\mathbf{W}_{1,1}$ & $W_{1,2}$ & $\mathbf{B}_{3,1}$ & $\mathbf{B}_{3,2}$ \\
\hline & $V_{2,1}$ & $\mathbf{V}_{2,2}$ & $\mathbf{V}_{2,3}$ & $W_{1,3}$ & $\mathbf{B}_{1, \mathbf{4}}$ & $\mathbf{B}_{3,3}$ & $\mathbf{B}_{3,2}$ \\
\hline \multirow{2}{*}{ بل الابناء } & $V_{1,1}$ & $V_{2,2}$ & $\mathbf{V}_{2,3}$ & $W_{1,1}$ & $B_{1,4}$ & $\mathbf{B}_{\mathbf{3 , 3}}$ & $\mathbf{B}_{3,2}$ \\
\hline & $V_{2,1}$ & $V_{1,2}$ & $V_{1,3}$ & $W_{1,3}$ & $W_{1,2}$ & $B_{3,1}$ & $\mathbf{B}_{3,2}$ \\
\hline
\end{tabular}

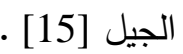

$$
\text { الثكل رقم (5): عبور نقطة البسيط }
$$

• الطفرة Mutation :هي المرحلة الأخيرة من سلسلة العمليات التكرارية التي تسهم بثكل جيد للوصول إلى الحل

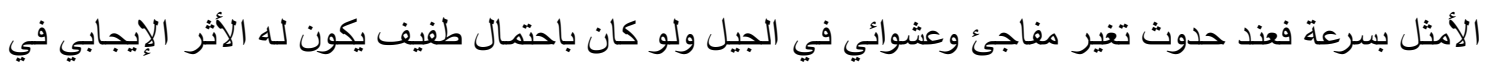

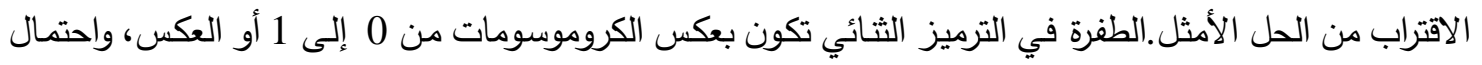
حدوث هذا التغير يعرف باحتمال الطفرة)Probability of Mutation (Pm وعادة يختار بقيمة متدنية نسبيًا [15]

6-2

الخوارزمية الجينية تتطلب وقتاً طويلاً لتتفيذها لكن في تقنيات الحاسوب الحديث تم التغلب على طول الوقت

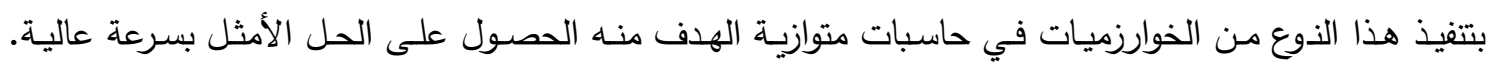

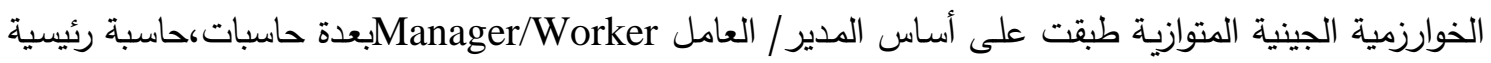
تعد المدير، و N من الحاسبات الطرفية بوصفها عمالا"، الموضحة بالثكل (6). المدير يعمل على تأسيس

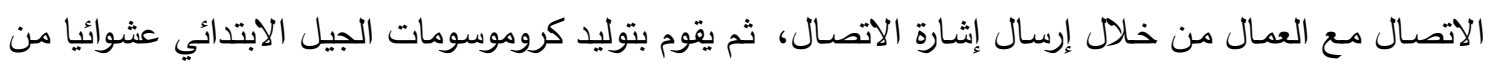

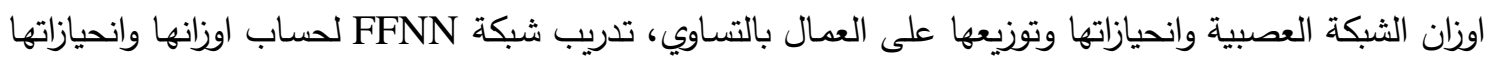

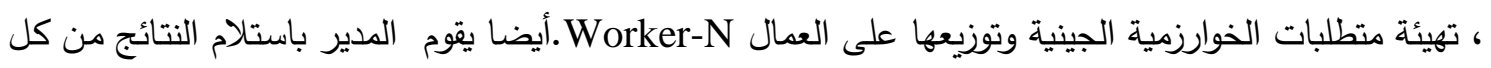
عامل لإعادة تمثيلها عشوائيا ليتسنى تكرار تلك العمليات، أرسال إثارة لانهاء الاتصال مع كل العمال بعد الحصول 
على الحل الأمثل النهائي. أما العمال N ، يقوم كل عامل من البداية باستلام الإثشارة من الددير لتأسيس الاتصال

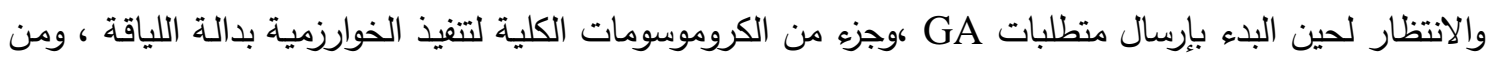

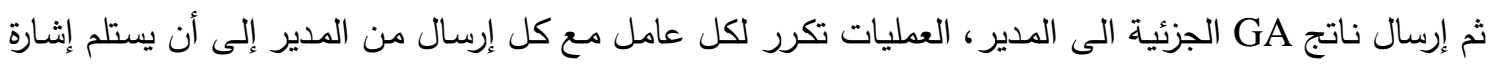
بإنهاء الاتصال بعد الوصول إلى الحل الأمثل النهائي [13][13] . الكسوري في حاسبات متعددة يوضح بالثكل (7).

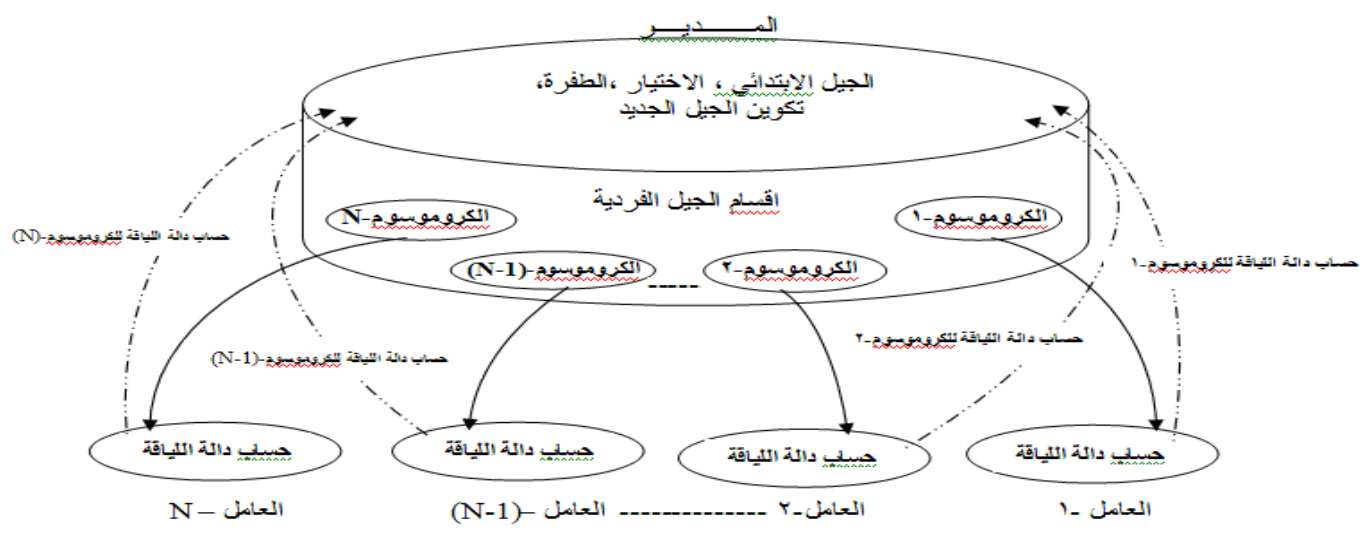

الثكل (6) الخوارزمية الجينية المتوازية]

3- مواد البحث وطرائقه :

استخدمت بيانات الصور الرمادية (Lena ,Barbara) هـ 8Bit/Pixel والذي يمثل شدة اللون الرمادي

بتدرجات (Girl,Butterfly) (0-255) ،والصور الملوني يتم فيها تخصيص ثلاث خانات لكل نقطة صورية

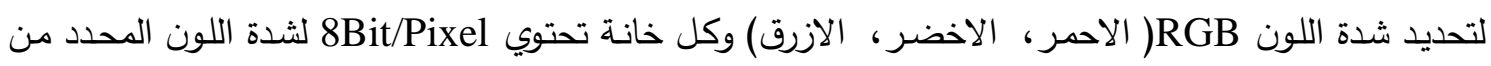

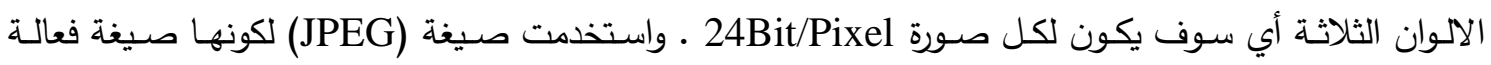
لضغط الصور الرقمية وهي الصيغة الاكثر شيوعا لحفظ وارسال ملفات الصور عبر شبكة الانترنت لان حجمها

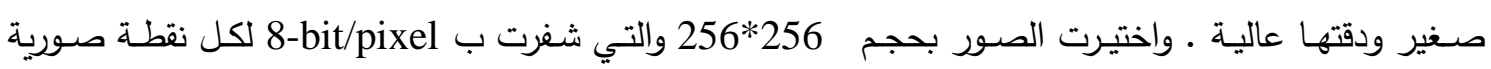

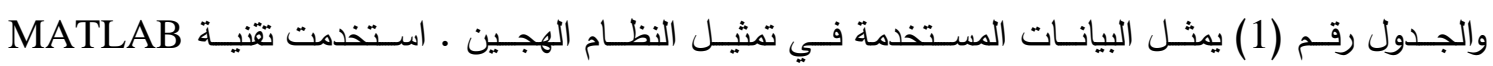
Distributed Computing Server (MDCS)

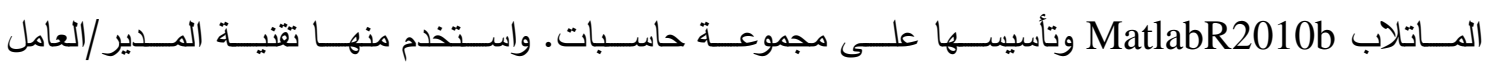
،يث ربطت (11) حاسبة ، واحدة منها تمثل المدير يعمل على إدارة النظام بوصفه خادماً

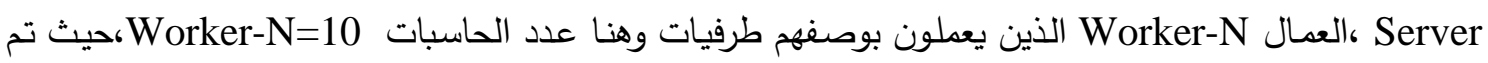

1-Install the MDCE Service on all computers تاسيس الاتصال كالاتي : n

2-In Dos for the folder:

Matlabrootldistcomp \bin\Mdce install

Matlabrootldistcomplbin $\backslash$ Mdce start

Matlabrootldistcomplbinladmincenter.bat

3-In admincenter: Create Job Manager : Jm1, Worker (1 - 10)

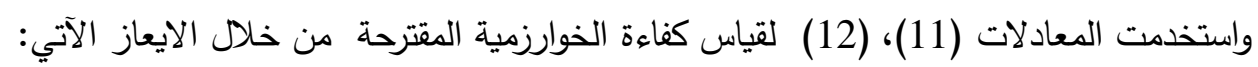

[PSNR,MSE,Error, Compression raio ] = measerr(image Name) 


$$
\begin{aligned}
& M S E=\frac{\sum_{i=0}^{M_{\text {Rows }}} \sum_{j=0}^{N_{\text {Cols }}}\left|X(i, j)-X_{c}(i, j)\right|^{2}}{M_{\text {Rows }} * N_{\text {Cols }}} \\
& P S N R=10 \log _{10}\left(\frac{255^{2}}{M S E}\right)
\end{aligned}
$$

\begin{tabular}{|c|c|c|c|}
\hline معادلة(9) & د الة اللياقة & 200,100 & حجم افراد الجيل الكلي المولد في المدير \\
\hline 50 & عدد الاجيال & 40,20 & حجم الجيل المستلم لكل عامل \\
\hline $4 * 4$ & حجم كتلة المدى & 1 & احتمالية العبور المثالية (Pc) \\
\hline 3 & عد العقد الخفية & 0.001 & احتمالية الطفرة المثالية (Pm) \\
\hline sigmoid AF & دالة التنشيط للطبقة الخفية & feedforwardNet & الثبكة العصبية الاصطناعية \\
\hline purline AF & دالة التشيط للاخراج & 2-3-1 & تصميم الثبكة العصبية \\
\hline
\end{tabular}

الجدول رقم (1) بيانات النظام الهجين (HPGANN)

4- - مناقشة النتائج :

تشير النتائج التي تم الحصول عليها بتطبيق النظام الهجين ان لله تأثيرا" في تحسين عملية الكبس وفك اك اكس الكبس الكسوري FIC وبسرعة أفضل من الطرائق (ANN,GA,FIC ) لكبس الصور الكسوري. تبين ذلك من

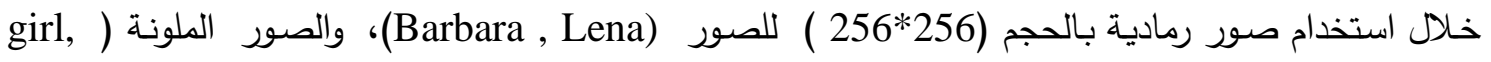
(Btterfly لنفس الحجم، وبشفرة 8-Bit/Pixel ، وخلال عملية التكرار لفك الكبس تم اخذ معدل النقاط الصورية الأربعة المتجاورة للصورة والتي تتجاوز المدى (0-255) لتجنب التباعد عن القيم الأصلية. النتائج قيست على أساس سرعة التتفيذ للنظام في حاسبات متعددة فعند اختيار حجم الجيل 200 ،التكرار 50 وبتنفيذات مختلفة يوضحها الجدول (2) للصور الرمادية كانت سرعة التتفيذ تتفاوت بزيادة عدد الحاسبات لصورة

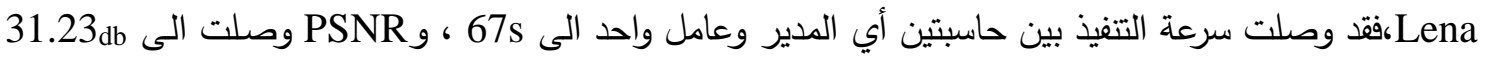

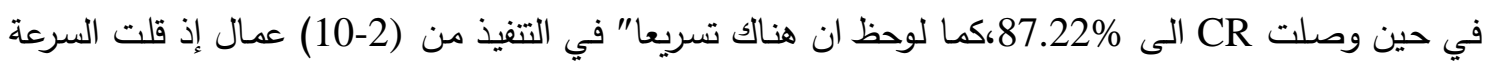

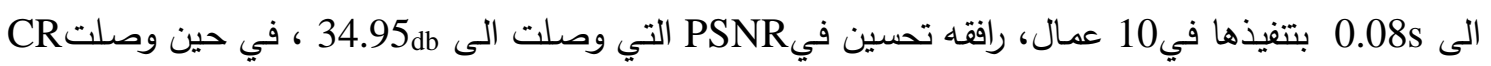
الى 91.56\%. كذلك لوحظ أن هناك تسريعاً في تنفيذ النظام عند تقليل حجم الجيل من فن 200 إلى 100 حيث حيث 100 حيث تراوح وقت التنفيذ بين s(65-0.02) لكل حالات التتفيذ الموضحة بالجدول (2)لصورة Lena ، رافقه أيضا ارتفاع

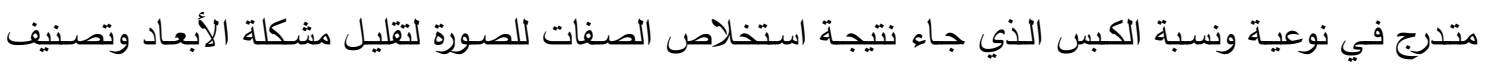

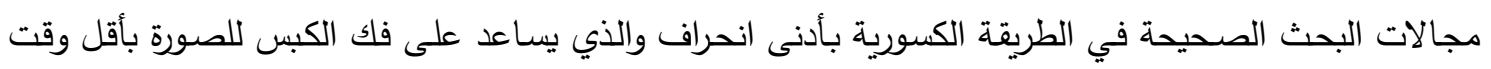

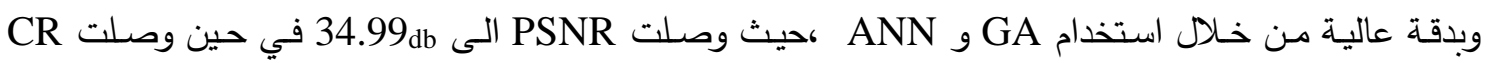
الى 91.43\% بأسرع تتفيذ للنظام وبعدد عمال مساوٍ لـ 10. 


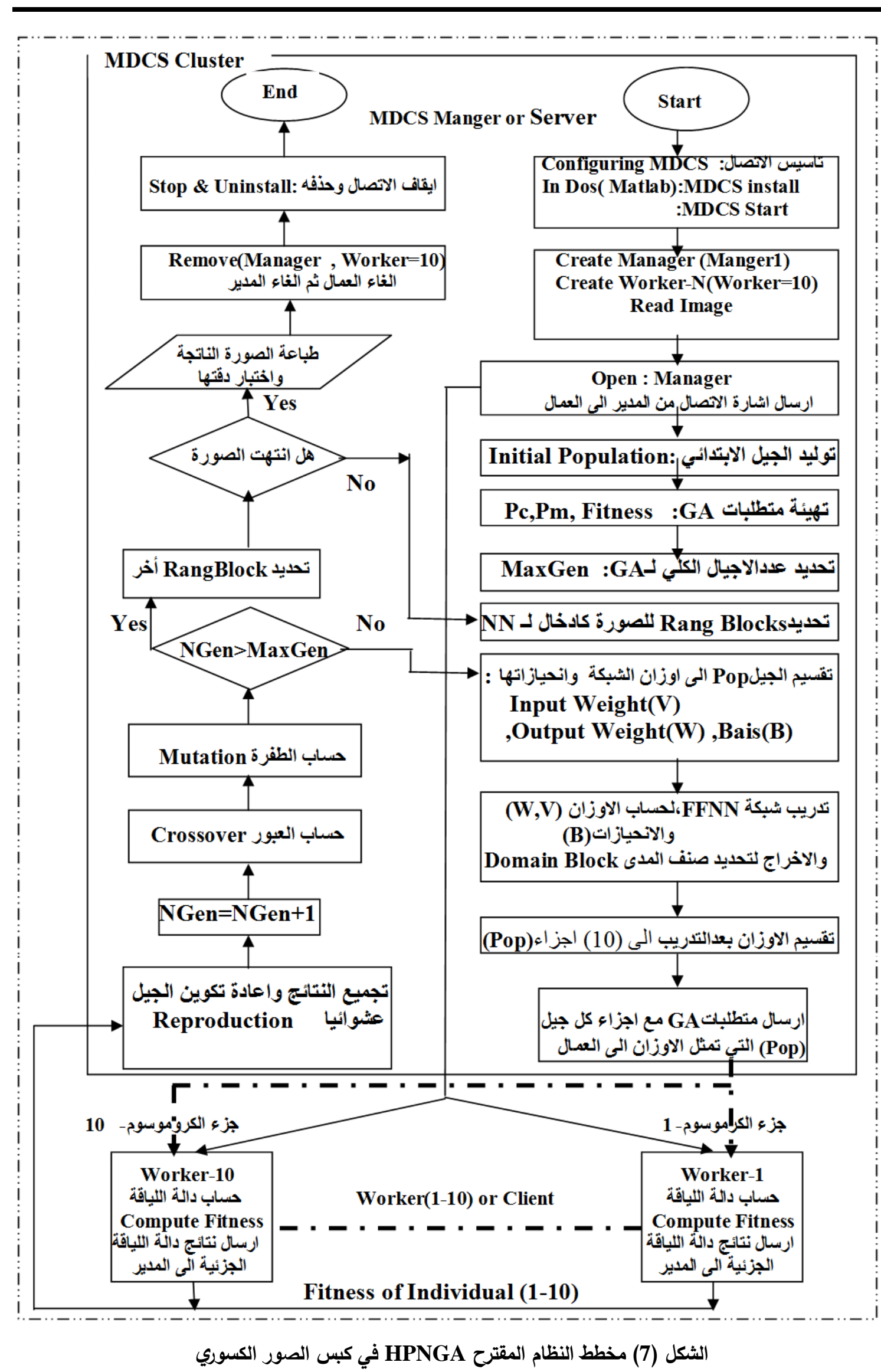


ولغـرض اختبار دقة النتائج التي طبقت على صـورة Lena تم تطبيق النظـام على الصـورة الرماديـة

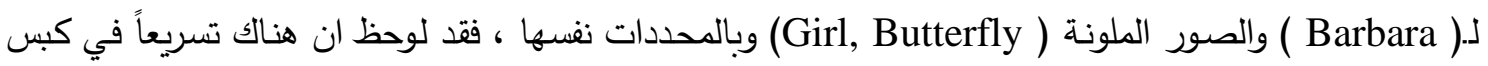
وفك الكبس باستخدام HPNGA يوضحها الجدول (2) والجدول (3) ، فعند اختيار حجم الجيل 200 وبتكرار 50 وال

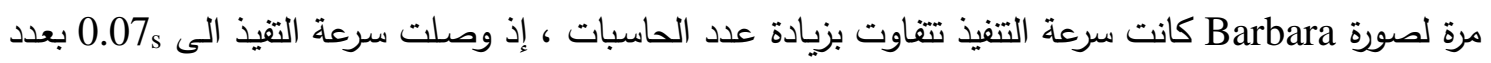

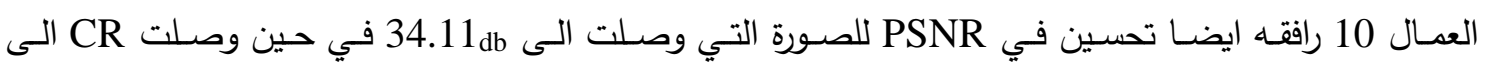
91.33\%. كذلك لوحظ ان هناك تسريعاً في تتفيذ النظام عند تقليل حجم الجيل الى 100 إذ وصلت سرعة التتفيذ

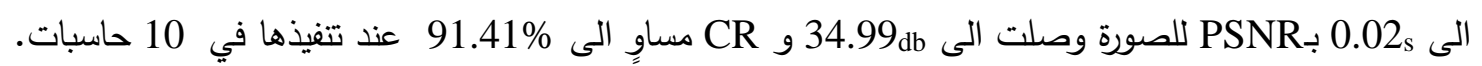
النتائج بينت ان هناك تسريعا في تتفيذ النظام الهجين للصور الملونة (Girl, Butterfly) عند تتفيذها في (10) حاسبات يوضحها الجدول (3) رغم الحجم المضاعف لها والذي كان بسعة 24-Bit/Pixelعن حجم الصور الرماديـة الذي كان بسعة 8-Bit/Pixel فـع اختلاف حجم الجيل من 200 الى 100 وبتكرار 50 مرة كانت سرعة التتفيذ تتفاوت بزيادة عدد الحاسبات للصورة Butterfly اذ وصلت الى s0.04 بعدد عمال 10 وحجم جيل

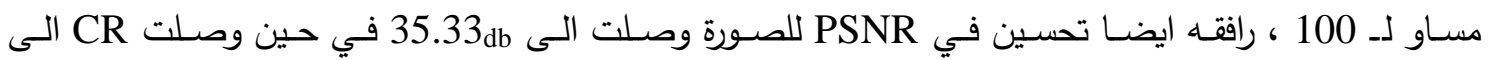
91.78\%. كذلك لوحظ ان سرعة التتفيذ تتفاوت بزيادة عدد الحاسبات للصورة Girl مع اختلاف حجم الجيل من وصن 200 الى 100 وبتكرار 50 مرة اذ وصلت الى s0.01 بعدد العمال 10 وحجم جيل مساو لـ 100 ، رافقه ايضـا

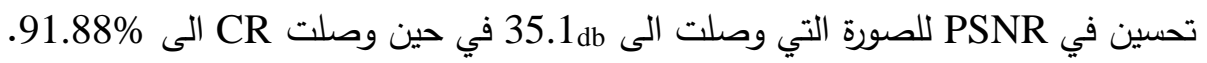
من الجداول (2) ,(3) يلاحظ انه مع بقاء التكرار ثابتاً حصل تسريع في تتفيذ النظام ووصل الى أفضل حالة بعدد عمال مساوٍ(10)، لكن مع انخفاض حجم الجيل من 200 الى 100 حصل تسريع أخرفي التتفيذ لكل

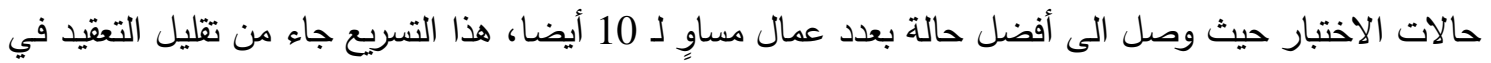
الحسابات لمشكلة الأبعاد في الطريقة الكسورية باستخدام ANN التي دمجت مع GA للوصول إلى القي القيم المثالية من الأوزان بانحيازاتها التي تصنف مجالات البحث الصحيحة بأدنى انحراف والذي يساعد على فلك الكبس للصور

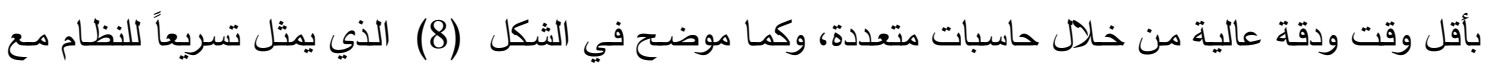

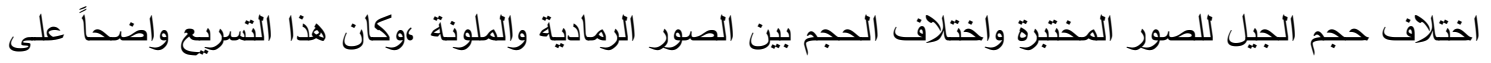
الصور بحصولها على نسبة ونوعية كبس عالية يوضحها الشكل (9) لصورة Lena التي طبقت بسرعة 0.02s، والثكل (10) لصورة Barbara التي طبقت بسرعة s0.02 ، والثكل (11) لصورة Butterfly التي طبقت بسرعةه0.04 والثكل(12) لصورة Girl التي طبقت بسرعة 0.01 . ولغرض قياس كفاءة النظـام الهجين تمت مقارنـة نتائجها مـع نتائج تتفيذ (GA،ANN،FIC) من خـلال تتفيذها في(10) حاسبات لنفس مواصفات النظام الهجين والموضحة بالجدول (4) إذ تبين ان تلك الطرائق أبطا في

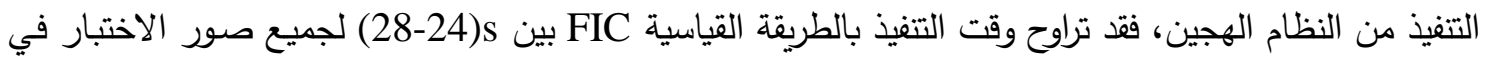
حين ترواحت PSNR بين db (30.38-30.1) ، و CR تراوحت بين \% (88.45-88.1). وحصل تسريع في التتفيذ

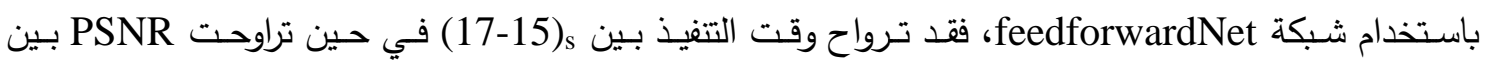

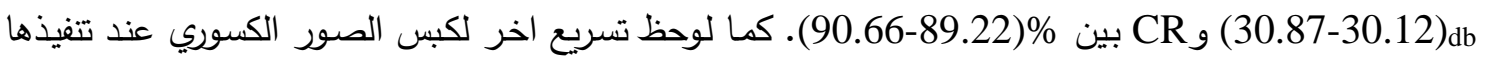

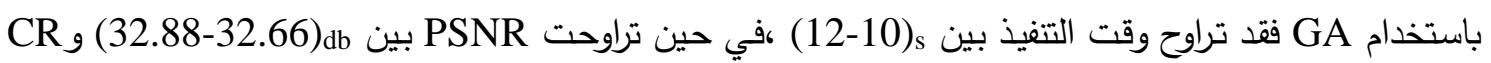
بين \% باسن (90.60-90.55). 
وحصل تسريع عالي لكبس الصور عند تطبيق النظام الهجين على صور الاختبار فقد تراوح وقت التتفيذ بين

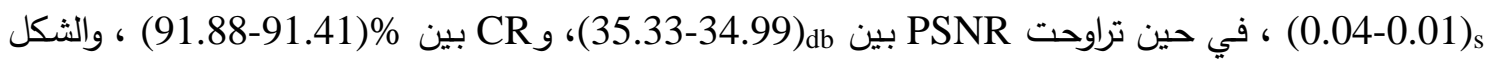
(13) يوضـح مقارنة النظام الهجين HPNGA مع الطرائق (GA,NN,FIC) .كما يوضح الثكل (14) لصورة Lena افضلية النظام الهجين في الحصول على كبس عالي الدقة للصور المختبرة ، فالصورة (b) وضحت الصورة الصورة بعد الكبس في النظام الهجين بوقت اقل من الصورة (c) الناتجة باستخدام الخوارزمية الكسورية القياسية(FIC)

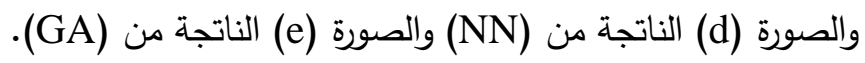
الجدول رقم (2) : سرعة التنفيذ في (10) حاسبات للصور الرمادية

\begin{tabular}{|c|c|c|c|c|c|c|c|c|}
\hline \multicolumn{3}{|c|}{ صورة (Barbara)/بحجم } & \multicolumn{3}{|c|}{ صورة (Lena)/256) بحجم } & \multicolumn{3}{|c|}{ الإدخالات } \\
\hline نسبة الكبس & $\begin{array}{c}\text { نوعية } \\
\text { PSNR }\end{array}$ & $\begin{array}{c}\text { الوقت } \\
\text { Tim } \\
\text { e }\end{array}$ & $\begin{array}{c}\text { نسبة } \\
\text { CR\% }\end{array}$ & $\begin{array}{c}\text { نوعية } \\
\text { PSNR }\end{array}$ & $\begin{array}{c}\text { الوقت } \\
\text { Tim } \\
\text { e }\end{array}$ & $\begin{array}{c}\text { التكرار } \\
\text { Iterati } \\
\text { on }\end{array}$ & $\begin{array}{c}\text { حجم الجيل } \\
\text { Population } \\
\text { size }\end{array}$ & $\begin{array}{c}\text { العمال } \\
\text { worke } \\
\text { rs } \\
\text { rs }\end{array}$ \\
\hline 87.21 & 31.27 & 66 & 87.22 & 31.23 & 67 & 50 & 200 & 1 \\
\hline 87.61 & 31.82 & 45 & 87.55 & 31.76 & 49 & 50 & 200 & 2 \\
\hline 88.46 & 32.28 & 30 & 88.42 & 32.21 & 31 & 50 & 200 & 3 \\
\hline 89.93 & 32.91 & 21 & 88.91 & 32.82 & 22 & 50 & 200 & 4 \\
\hline 90.23 & 33.42 & 18 & 90.22 & 33.28 & 19 & 50 & 200 & 5 \\
\hline 90.36 & 33.56 & 12 & 90.44 & 33.35 & 12 & 50 & 200 & 6 \\
\hline 90.65 & 33.78 & 4 & 90.86 & 33.55 & 3 & 50 & 200 & 7 \\
\hline 90.88 & 33.89 & 2 & 91.22 & 34.57 & 2 & 50 & 200 & 8 \\
\hline 91.12 & 33.94 & 0.6 & 91.34 & 34.90 & 0.5 & 50 & 200 & 9 \\
\hline 91.33 & 34.11 & 0.07 & 91.56 & 34.95 & 0.08 & 50 & 200 & 10 \\
\hline 88.51 & 31.41 & 63 & 88.53 & 31.35 & 65 & 50 & 100 & 1 \\
\hline 88.92 & 31.95 & 41 & 88.92 & 31.89 & 43 & 50 & 100 & 2 \\
\hline 89.56 & 32.91 & 26 & 89.54 & 32.87 & 28 & 50 & 100 & 3 \\
\hline 90.23 & 33.95 & 19 & 90.2 & 33.93 & 20 & 50 & 100 & 4 \\
\hline 90.55 & 34.42 & 16 & 90.53 & 34.33 & 17 & 50 & 100 & 5 \\
\hline 90.66 & 34.65 & 10 & 90.72 & 34.42 & 10 & 50 & 100 & 6 \\
\hline 90.87 & 34.71 & 3 & 90.92 & 34.61 & 2 & 50 & 100 & 7 \\
\hline 91.1 & 34.82 & 0.9 & 91.11 & 34.77 & 0.6 & 50 & 100 & 8 \\
\hline 91.23 & 34.91 & 0.08 & 91.24 & 34.85 & 0.2 & 50 & 100 & 9 \\
\hline 91.41 & 34.99 & 0.02 & 91.43 & 34.99 & 0.02 & 50 & 100 & 10 \\
\hline
\end{tabular}

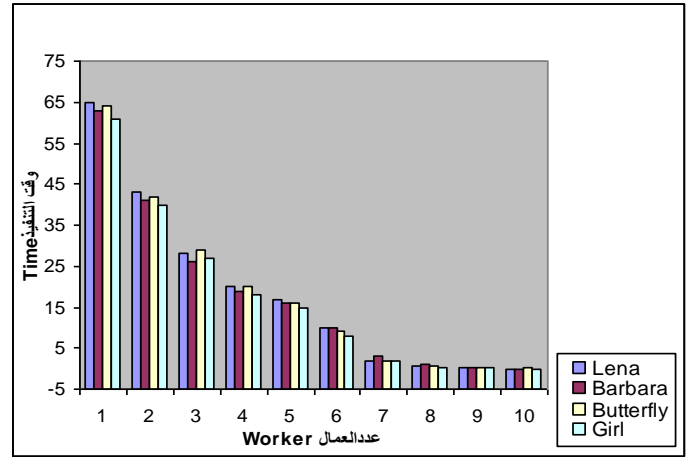

(100) تسريع الصور المختبرة بحجم الجيل(B)

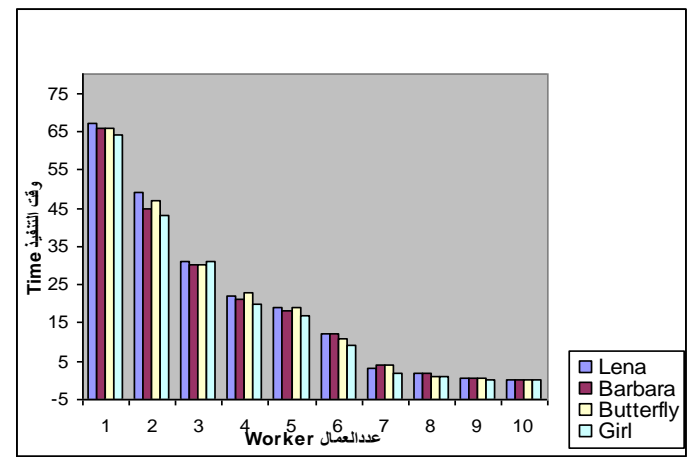

(200) تسريع الصور المختبرة بحجم الجيل(A) الثكل (8) تسريع النظام الهجين (HPNGA) في كبس الصور الكسوري 
الجدول رقم (3) :سرعة التنفيذ في (10) حاسبات للصور الملونة

\begin{tabular}{|c|c|c|c|c|c|c|c|c|}
\hline \multicolumn{3}{|c|}{ صورة (Girl) / بجم (256*256) } & \multicolumn{3}{|c|}{ صورة ( Butterfly) / صجم (256"256) } & \multicolumn{3}{|c|}{ الادخالات } \\
\hline $\begin{array}{c}\text { الكبس } \\
\text { CR\% }\end{array}$ & $\begin{array}{c}\text { نوعية } \\
\text { الكبس } \\
\text { PSNR }\end{array}$ & $\begin{array}{c}\text { الوقت } \\
\text { Time }\end{array}$ & $\begin{array}{c}\text { نسبة } \\
\text { الكبس } \\
\text { CR\% }\end{array}$ & $\begin{array}{c}\text { نوعية } \\
\text { الكبس } \\
\text { PSNR }\end{array}$ & الوقت & $\begin{array}{c}\text { التكرار } \\
\text { Iteration }\end{array}$ & $\begin{array}{l}\text { حجم الجيل } \\
\text { Population } \\
\text { size }\end{array}$ & $\begin{array}{l}\text { عددالعمال } \\
\text { workers }\end{array}$ \\
\hline 88.67 & 31.28 & 64 & 87.41 & 31.25 & 66 & 50 & 200 & 1 \\
\hline 88.87 & 31.81 & 43 & 87.56 & 31.77 & 47 & 50 & 200 & 2 \\
\hline 89.52 & 32.31 & 31 & 88.45 & 32.28 & 30 & 50 & 200 & 3 \\
\hline 89.91 & 32.92 & 20 & 88.92 & 32.91 & 23 & 50 & 200 & 4 \\
\hline 90.33 & 33.52 & 17 & 90.93 & 33.35 & 19 & 50 & 200 & 5 \\
\hline 90.56 & 33.69 & 9 & 91.12 & 33.62 & 11 & 50 & 200 & 6 \\
\hline 90.88 & 33.88 & 2 & 91.23 & 33.85 & 4 & 50 & 200 & 7 \\
\hline 91.12 & 33.95 & 1 & 91.44 & 33.98 & 1 & 50 & 200 & 8 \\
\hline 91.34 & 34.16 & 0.2 & 91.65 & 34.22 & 0.5 & 50 & 200 & 9 \\
\hline 91.78 & 34.58 & 0.03 & 91.84 & 34.54 & 0.06 & 50 & 200 & 10 \\
\hline 88.61 & 31.42 & 61 & 88.54 & 31.41 & 64 & 50 & 100 & 1 \\
\hline 89.22 & 32.21 & 40 & 88.93 & 31.87 & 42 & 50 & 100 & 2 \\
\hline 89.72 & 33.11 & 27 & 89.53 & 32.81 & 29 & 50 & 100 & 3 \\
\hline 90.32 & 33.97 & 18 & 90.23 & 33.91 & 20 & 50 & 100 & 4 \\
\hline 90.62 & 34.71 & 15 & 90.61 & 34.41 & 16 & 50 & 100 & 5 \\
\hline 90.91 & 34.75 & 8 & 90.89 & 34.61 & 9 & 50 & 100 & 6 \\
\hline 91.37 & 34.88 & 2 & 91.25 & 34.83 & 2 & 50 & 100 & 7 \\
\hline 91.44 & 34.91 & 0.3 & 91.39 & 34.93 & 0.7 & 50 & 100 & 8 \\
\hline 91.69 & 34.99 & 0.08 & 91.56 & 35.13 & 0.1 & 50 & 100 & 9 \\
\hline 91.88 & 35.1 & 0.01 & 91.78 & 35.33 & 0.04 & 50 & 100 & 10 \\
\hline
\end{tabular}

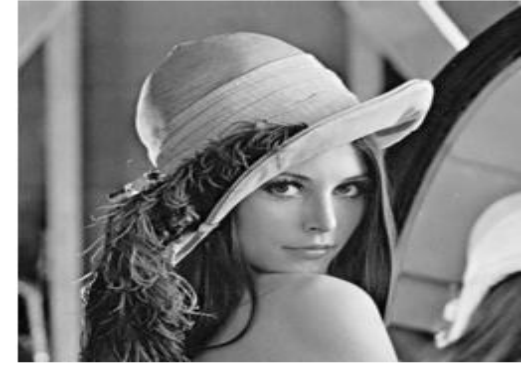

(b) (الصورة بعد الكبس (b)

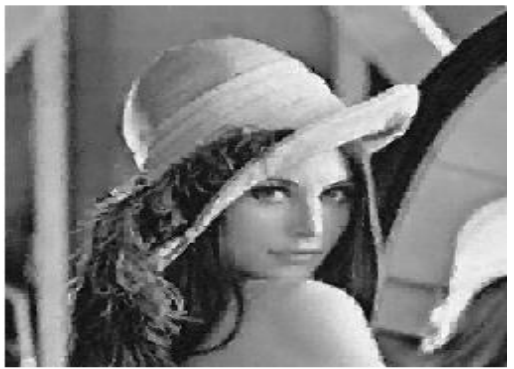

(a) (a) (الصورة الاصلية

الثكل (9) صورة Lena الناتجة من النظام الهجين بسرعة 0.02

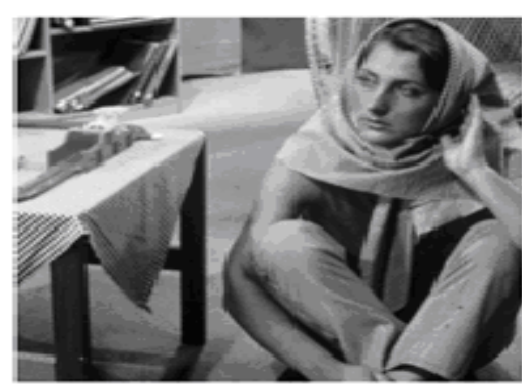

(b) (الصورة بعد الكبس (b)

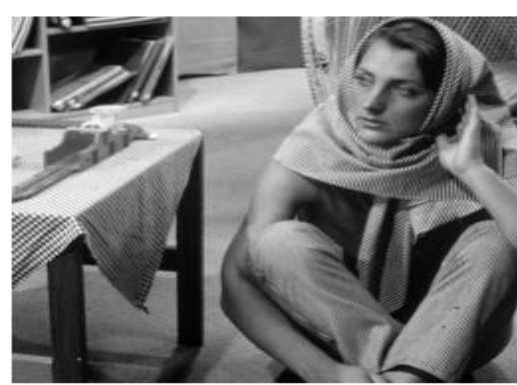

(a) (a) (الصورة الاصلية

الثكل (10) صورة Barbara الناتجة من النظام الهجين بسرعة، 


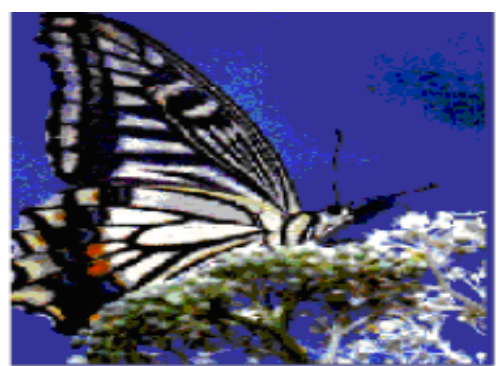

(b) (b) الصورة بعد الكبس

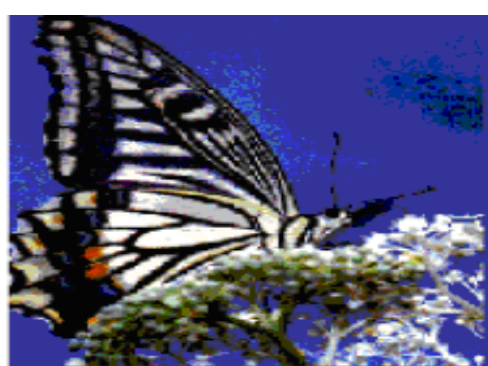

(a) (a) (a) (الصورة الاصلية

الثكل (11) صورة Butterfly الناتجة من النظام الهجين بسرعةs0.04

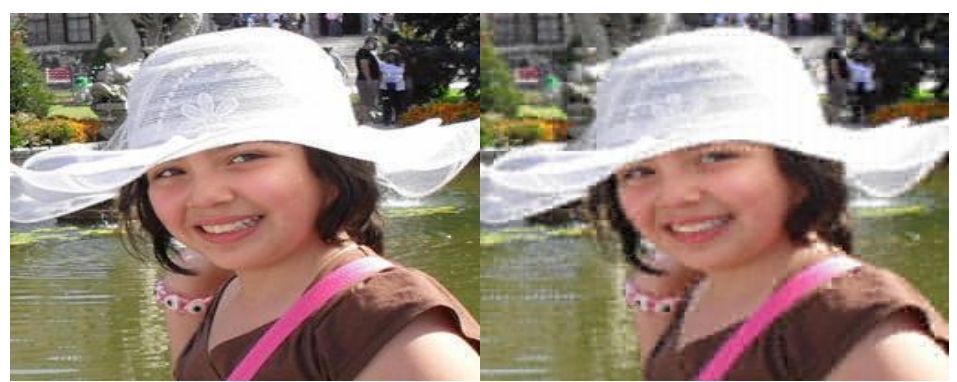

(b) (الصورة بعد الكبس

(a) (a) (الصورة الاصلية

الثكل (12) صورة Girl الناتجة من النظام الهجين بسرعة،0.01

الجدول رقم (4) : مقارنة HPNGA مع الطرائق (GA,NN,FIC)

\begin{tabular}{|c|c|c|c|c|}
\hline \multicolumn{5}{|c|}{ Time(S) الوقت } \\
\hline & FIC & NN & GA & HPNGA \\
\hline Lena & 28 & 15 & 10 & 0.02 \\
\hline Barbara & 25 & 17 & 11 & 0.02 \\
\hline Butterfly & 24 & 16 & 11 & 0.04 \\
\hline Girl & 27 & 15 & 12 & 0.01 \\
\hline \multicolumn{5}{|c|}{ نوعية الكبس (PSNR )db ) } \\
\hline & FIC & NN & GA & HPNGA \\
\hline Lena & 30.1 & 30.12 & 32.66 & 34.99 \\
\hline Barbara & 30.2 & 30.17 & 32.78 & 34.99 \\
\hline Butterfly & 30.24 & 31.67 & 32.65 & 35.33 \\
\hline Girl & 30.38 & 31.87 & 32.88 & 35.1 \\
\hline \multicolumn{5}{|c|}{ نسبة الكبس ( Compression ratio\%) } \\
\hline & FIC & NN & GA & $\overline{\text { HPNGA }}$ \\
\hline Lena & 88.1 & 89.22 & 90.55 & 91.43 \\
\hline Barbara & 88.21 & 89.27 & 90.61 & 91.41 \\
\hline Butterfly & 88.34 & 89.61 & 90.76 & 91.78 \\
\hline Girl & 88.45 & 90.66 & 90.65 & 91.88 \\
\hline
\end{tabular}




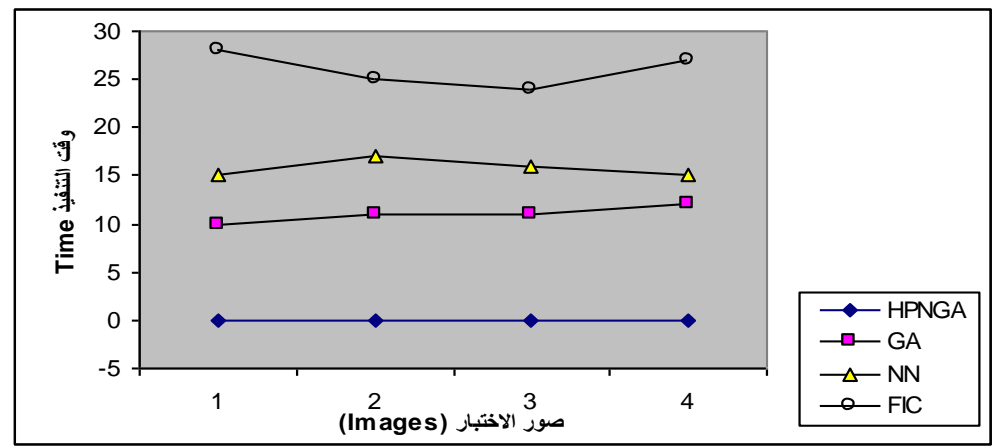

الثكل (13) مقارنة سرعة HPNGA مع سرعة الطرائق (GA,NN,FIC)

5- الاستنتاجات والتوصيات

النظام الهجين HPNGA أثبت نجاحه في تحسين كبس الصور الكسوري من خلال استخلاص صفات الصور لتقليل مشكلة الابعاد للصور الرمادية والملونـة ومكنت ANN من تدريب الصور والحصول على الاوزان المثالية باستخدام GA التي تصنف مجال البحث بادنى انحراف الذي يساعد على كبس الصور الكسوري بدقة

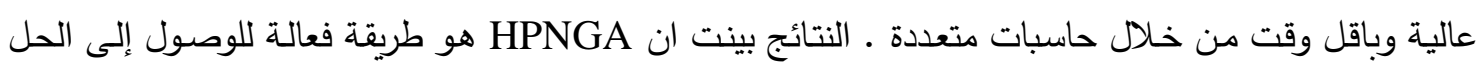

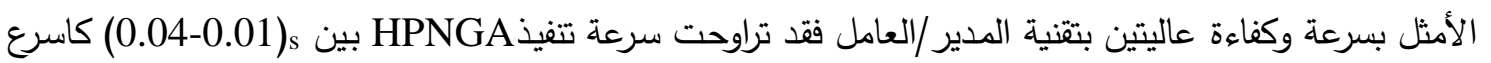
تتفيذ لكبس الصور الكسوري في (11) حاسبة,وان طريقة استخلاص الصفات المستخدمة للكبس وفك الكبس،

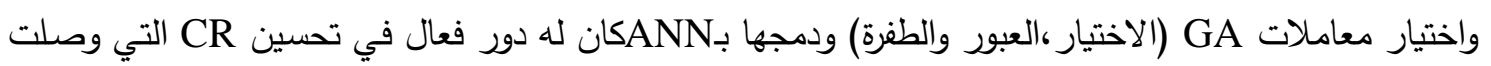

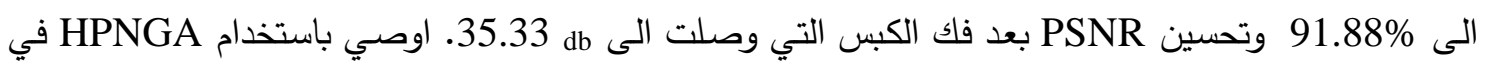
ايجاد الحلول لاي مشكلة اذا كان عامل السرعة مهماً لحلها خاصة تلك المشاكل التي تحل باستخدام GA.
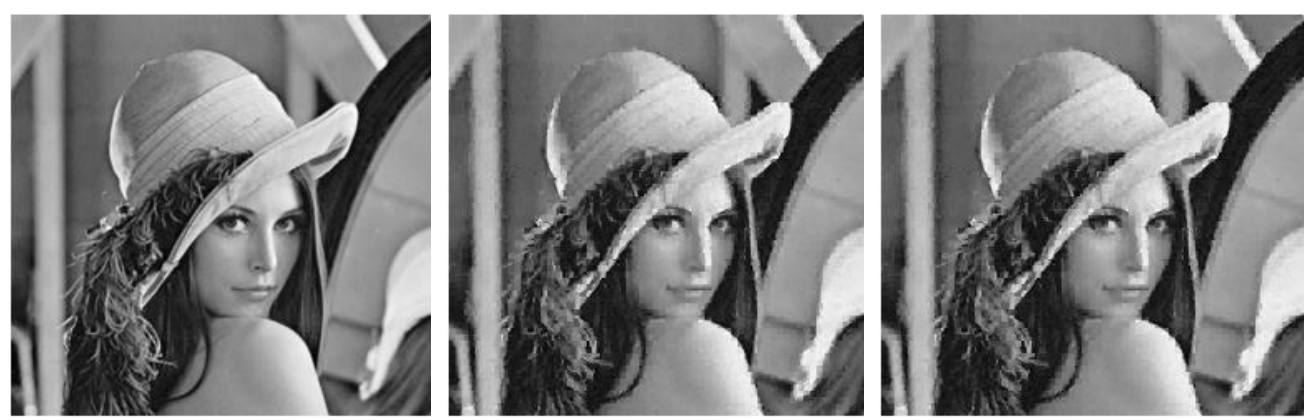
القياسية FIC (c)
HPNGA باستخدام FIC (b)
الصورة الأصلية
(a) الثكل

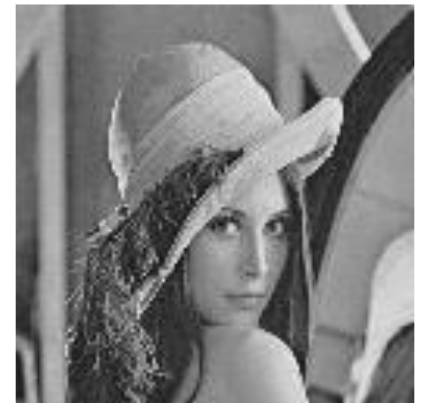

GA باستخذامFIC (e)

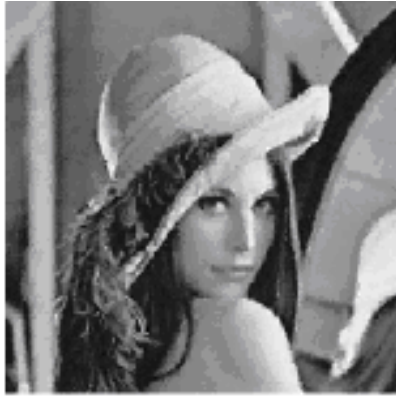

NN باستخدام FIC (d)

الثكل (14) مقارنة نتائج الخوارمية المقترحة HPNGA مع الطرائق(GA,NN,FIC) 


\section{المصادر}

[1] Mingshui Li, Shanhu Ou and Heng Zhang: The new progress in research approach of fractal image compression. Journal of Engineering Graphics. 4(3), 143-152, 2004.

[2] S. N. Sivanandam, S. Sumathi, S. N. Deepa, "Introduction To Neural Networks Using Matlab 6.0", Tata McGraw-Hill, 2006.

[3] M. Mohamed, "Optimization Of Fractal Image Compression Based On Kohonen Neural Networks", EEDIS Laboratory, Engineering Faculty, University of SBA, 2006.

[4] A. Ben, M. Ben and Y. Ben, "Optimization of fractal image compression with genetic algorithms", A. Ben Jmaa is with National School of Engineering of Sfax, BP, W, 3038 Sfax, 2006.

[5] X. Lifeng, Z. Liangbin, "A Study of Fractal Image Compression Based on an Improved Genetic Algorithim", Zhejiang Wanli University, 2007.

[6] Y. Chakrapani, K. Soundara, "Genetic Algorithm Applied To Fractal Image Compression", Dept. of Electronic and communications, J.N.T.U College of Engineering, India, 2009.

[7] M.Sheng, J.H. Jeng, "Schema genetic algorithm for Fractal Image Compression", Dept. of Electronic Engineering, N.S. Yet-Sen University, Taiwan, 2006.

[8] B.S. Sanjay, M.V. Kulkarni, "Fractal Image Compression Using Genetic Algorithim", Advances in computer Engineering ACE, India, June 2010.

[9] Y. Chakrapani, K. Soundara, "A Comparative Approach To Fractal Image Compression Using Genetic Algorithm and Simulated Annealing Technique", Dept. of Electronic and communications, J.N.T.U College of Engineering, India, 2008.

[10] Y. Chakrapani, K. Soundara, "Adaptive Neuro-Fuzzy Inference System based Fractal Image Compression", Dept. of Electronic and communications, J.N.T.U College of Engineering, India,Vol 2, No.1, November, 2009.

[11] Y. Chakrapani, K. Soundara, "Hybrid Genetic -Simulated Annealing Approach for Fractal Image Compression", Dept. of Electronic and communications, J.N.T.U College of Engineering, India, 2008.

[12] Y. Chakrapani, K. Soundara, "Implementation of Fractal Image Compression Hybrid Genetic -Neural Approach", Dept. of Electronic and communications, J.N.T.U College of Engineering, India,Vol.7, No. 3, September 2009.

[13] P. Borovska, "Solving the Travelling Salesman Problem in Parallel By Genetic Algorithm on Multicomputer Cluster", Computer Systems Dept., Technical University of Sofia, 2006.

[14] S.Tongchim, Prabhas, "Parallel Genetic Algorithm for Finite-State Machine Synthesis From Input/Output Sequences", Department of Computer Engineering, Chulalongkorn University Bangkok 10330, Thailand, 2000. 
[15] الحجار ، م .عبيد، م، "الخوارزميات الجينية"، إشراف العرفي، هادي، مشروع دبلوم، جامعة دمشق، كلية

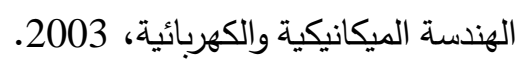

[16] الجار الله، م. الحربي، ن، "الحاسبات المتوازية والخوارزميات المتوازية"، جامعة الملك سعود، كلية العلوم،

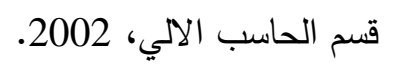

